ARTICLE

\title{
Proteogenomics analysis unveils a TFG-RET gene fusion and druggable targets in papillary thyroid carcinomas
}

Aswini Krishnan 1,11, Jean Berthelet ${ }^{1,11}$, Emilie Renaud', Sebastian Rosigkeit', Ute Distler (1) 2, Eric Stawiski ${ }^{3}$, Jing Wang ${ }^{3}$, Zora Modrusan ${ }^{4}$, Marc Fiedler (10 5, Mariann Bienz (10 5, Stefan Tenzer (1) ${ }^{2}$, Arno Schad ${ }^{6}$, Wilfried Roth ${ }^{6}$, Bernd Thiede (iD ${ }^{7}$, Somasekar Seshagiri (iD ${ }^{4,8}$, Thomas J. Musholt ${ }^{9}$ \&

Krishnaraj Rajalingam (iD 1,10凶

Papillary thyroid cancer (PTC) is the most common type of endocrine malignancy. By RNAseq analysis, we identify a RET rearrangement in the tumour material of a patient who does not harbour any known RAS or BRAF mutations. This new gene fusion involves exons 1-4 from the 5' end of the Trk fused Gene (TFG) fused to the 3' end of RET tyrosine kinase leading to a TFG-RET fusion which transforms immortalized human thyroid cells in a kinasedependent manner. TFG-RET oligomerises in a PB1 domain-dependent manner and oligomerisation of TFG-RET is required for oncogenic transformation. Quantitative proteomic analysis reveals the upregulation of E3 Ubiquitin ligase HUWE1 and DUBs like USP9X and UBP7 in both tumor and metastatic lesions, which is further confirmed in additional patients. Expression of TFG-RET leads to the upregulation of HUWE1 and inhibition of HUWE1 significantly reduces RET-mediated oncogenesis.

\footnotetext{
${ }^{1}$ Cell Biology Unit, University Medical Center of the Johannes Gutenberg University Mainz, 55131 Mainz, Germany. ${ }^{2}$ Institute of Immunology, University Medical Center of the Johannes Gutenberg University Mainz, 55131 Mainz, Germany. ${ }^{3}$ MedGenome, Inc., Foster City, CA, USA. ${ }^{4}$ Molecular Biology Department, Genentech, Inc., South San Francisco, CA, USA. ${ }^{5}$ MRC Laboratory of Molecular Biology, Cambridge Biomedical Campus, Francis Crick Avenue, Cambridge $\mathrm{CB} 2 \mathrm{OQH}, \mathrm{UK} .{ }^{6}$ Institute of Pathology, University Medical Center Mainz, Mainz, Germany. ${ }^{7}$ Department of Biosciences, University of Oslo, 0371 Oslo, Norway. ${ }^{8}$ SciGenom Research Foundation, Bangalore, India. ${ }^{9}$ Endocrine Surgery Section, Department of General Visceral and Transplantation Surgery, University Medicine, Mainz, Germany. ${ }^{10}$ University Cancer Center Mainz, University Medical Center, Mainz, Germany. ${ }^{11}$ These authors contributed equally: Aswini Krishnan, Jean Berthelet ${ }^{{ }}$email: krishna@uni-mainz.de
} 
$\mathrm{P}$ apillary thyroid carcinoma (PTC) is one of the most common form of thyroid cancer. A recent survey by Surveillance, Epidemiology, and End Results (SEER) program estimates 53,990 new cases of thyroid cancer and 2060 deaths in the US, which would account for $3.1 \%$ of overall cancer cases and $0.3 \%$ of all cancer deaths in $2018^{1,2}$. Although the death rate from thyroid cancer is low, its incidence increased annually by $3.6 \%$ from 1974 to 2013 and it is the most rapidly increasing cancer in the US ${ }^{3}$. Thyroid cancer occurs three times more frequently in women than in men, though studies indicate that male patients are presented with more aggressive stages when diagnosed and have lower disease free survival as well as higher mortality ${ }^{4,5}$. Nearly $80 \%$ of all thyroid cancers are $\mathrm{PTCs}^{6}$. Other major types of thyroid cancer include follicular thyroid cancer (FTC), anaplastic thyroid cancer (ATC) and medullary thyroid cancer (MTC), and the distinction is primarily based on the cell of origin and tissue architecture ${ }^{7}$. Thus far, surgery and radioiodine treatment (RAI) represent the major therapeutic avenue for patients ${ }^{8}$. Even though patients with PTCs mostly have a good prognosis, recurrence and aggressive metastases have been found in an increasing number of patients in the last years ${ }^{9}$. Central lymph node (LN) metastases are often detected in $20-90 \%$ PTC patients and considered as a factor contributing to recurrence and morbidity in a study employing a large cohort of patients ${ }^{10}$. The BRAF mutation resulting in its highly kinase active protein form BRAFV600E and mutations in the RAS gene, especially NRAS, are the most common genetic alterations seen in $\mathrm{PTC}^{7}$. Apart from $B R A F$ and NRAS mutations, common genetic alterations in PTCs include gene fusions involving the RET gene giving rise to oncogenic fusion proteins that account for up to $13-25 \%$ of PTCs ${ }^{7,11}$. Although BRAF mutations are prevalent in older patients, RET fusions are much more frequent in younger patients. RET fusions (also called RET/PTC rearrangements) are genomic rearrangements that are associated with ionizing radiation-induced DNA damage. RET fusions were reported in up to $60 \%$ cases of post Chernobyl PTCs ${ }^{12}$. Spatial contiguity of the genes involved in the fusion during interphase could be the structural basis of these chromosomal rearrangements ${ }^{13}$. In oncogenic RET rearrangements the kinase domain-containing $\mathrm{C}$ terminus of the RET gene, which is normally not expressed in thyroid follicular cells, is fused to the promoter-containing $\mathrm{N}$ terminus of a ubiquitously expressed, unrelated gene $\mathrm{e}^{14}$.

In this study, we aimed at the identification and characterization of the molecular events underlying PTC. By employing proteogenomic analysis of matching normal vs tumor vs lymph node metastasis of the same patient, we identified and validated a novel oncogenic RET fusion as well as other druggable targets in PTCs. We extended our proteomics observations by analyzing a cohort of PTC patient samples. Further, we provide mechanistic insights on the activation of the TFG-RET fusion and identified that E3 ubiquitin ligase HUWE1 is required for RET-mediated oncogenic transformation.

\section{Results}

Identification of a novel oncogenic RET fusion in a PTC patient. From a cohort of PTC patients who are devoid of RAS and BRAFV600E mutations, a single patient who had a tumor mass largely in the right thyroid with multiple lateral lymph node metastases was selected. Tumor and LN metastatic tissue were harvested intraoperatively according to institutional guidelines with due ethical consent. Normal thyroid tissue from the left thyroid lobe was harvested during operation and served as a matching control. Histopathological analysis was performed to confirm the tumor content and the tissue specificity (Fig. 1a). In addition, $\alpha$-calcitonin was detected in some cells of the primary tumor tissue implying c-cell hyperplasia and there was no $a$-calcitonin expression in the LN metastatic tissue (Fig. 1a). The tissue was lysed following a standard operating procedure as mentioned in the Methods section to collect the DNA, RNA and protein samples for subsequent genomics and proteomics analysis.

We next examined genomic alterations using both RNA-seq and exome-seq of the normal, tumor and LN metastasis samples. Exome data analysis of normal vs tumor and normal vs LN metastasis showed a total of 14 gene mutations of which 6 were shared between the tumor and LN metastasis (Supplementary Fig. 1A, B, Table 1). None of these mutations were well characterized mutations in known oncogenes or tumor suppressors. Recent studies suggest that more than $70 \%$ of PTCs harboractivating mutations in BRAF, NRAS or HRAS ${ }^{15}$. We next examined RNA-seq data to look for other potential genomic drivers. RNA-seq-based fusion analysis detected a rearrangement where the $5^{\prime}$ end of the Trk fusion gene (TFG), which carries a PB1 domain, is fused to the $3^{\prime}$ kinase domain of the RET tyrosine kinase leading to a novel RET fusion (Fig. 1b). RET fusions involving other members have been characterized previously and occur in $7 \%$ of PTCs ${ }^{15,16}$. The fusion junction reads were detected only in the tumor and metastatic sample, thus confirming that the identified gene fusion is a somatic and not a germline event (Supplementary Fig. 1D, Supplementary Data 1). Differential expression analysis between the tumor and matched normal revealed 244 significantly deregulated genes ( $q$-value $<0.001$, Supplementary Data 2 and 3). Among these, we identified RET kinase as being significantly upregulated (Fig. 1c, Supplementary Fig. 1E). RET expression was at 14.7 and 10.2 RPMK in the LN metastatic and tumor sample, respectively, compared to 0.3 RPKM in the adjacent normal (Supplementary Fig. 1F). The average RET expression in normal thyroid tissue from GTEX (http://gtexportal.org) is 0.3 RPKM (Supplementary Fig. 1G). We examined previously described RET fusions in PTCs and found that RET fusions lead to significant overexpression of the RET gene (Supplementary Fig. $1 \mathrm{H}, p$-value $<0.00001)^{15}$. Further, we isolated the mRNA from the patient's tumor lesion to generate cDNA, which was subjected to Sanger sequencing using primers covering the fusion region to confirm the presence of the fusion transcript in the tumor but not in the matching normal tissue (Supplementary Fig. 2A-C).

We then aimed to characterize the potential oncogenic activity of the RET fusion by stably expressing this gene fusion in immortalized human thyroid Nthy-ori 3-1 cells (from now on referred to as Nthy-TFG-RET cells). As expected, stable expression of TFG-RET fusion lead to increased viability (MTT assay) and cell proliferation (EdU assay) of Nthy-ori 3-1 cells (Fig. 2a, b and Supplementary Fig. 3A). Stable expression of TFGRET cells lead to the transformation of these cells as revealed by soft agar colony formation assays (Fig. 2c). Further, TFG-RET expression activated several downstream pro-survival signaling pathways (Fig. 2d and Supplementary Fig. 3B). Our next step was to investigate whether TFG-RET expression could enable tumor formation in vivo. Subcutaneous injection of Nthy-TFG-RET cells into NOD/SCID mice, after a latency of about 12 weeks, showed tumor formation in mice carrying Nthy-TFG-RET cells, while the control group did not exhibit any tumor growth (Fig. 2e-g). Over the latency period, one shall not rule out the possibility that the Nthy-TFG-RET cells might have acquired additional mutations, which ultimately lead to tumor growth. However, the lack of tumor growth in the control group validates the oncogenic potential of TFG-RET. Together, these assays establish TFG-RET fusion as a thyroid oncogene.

Characterization of TFG-RET. In vitro kinase assays showed that the TFG-RET fusion exhibits constitutive kinase activity 
a

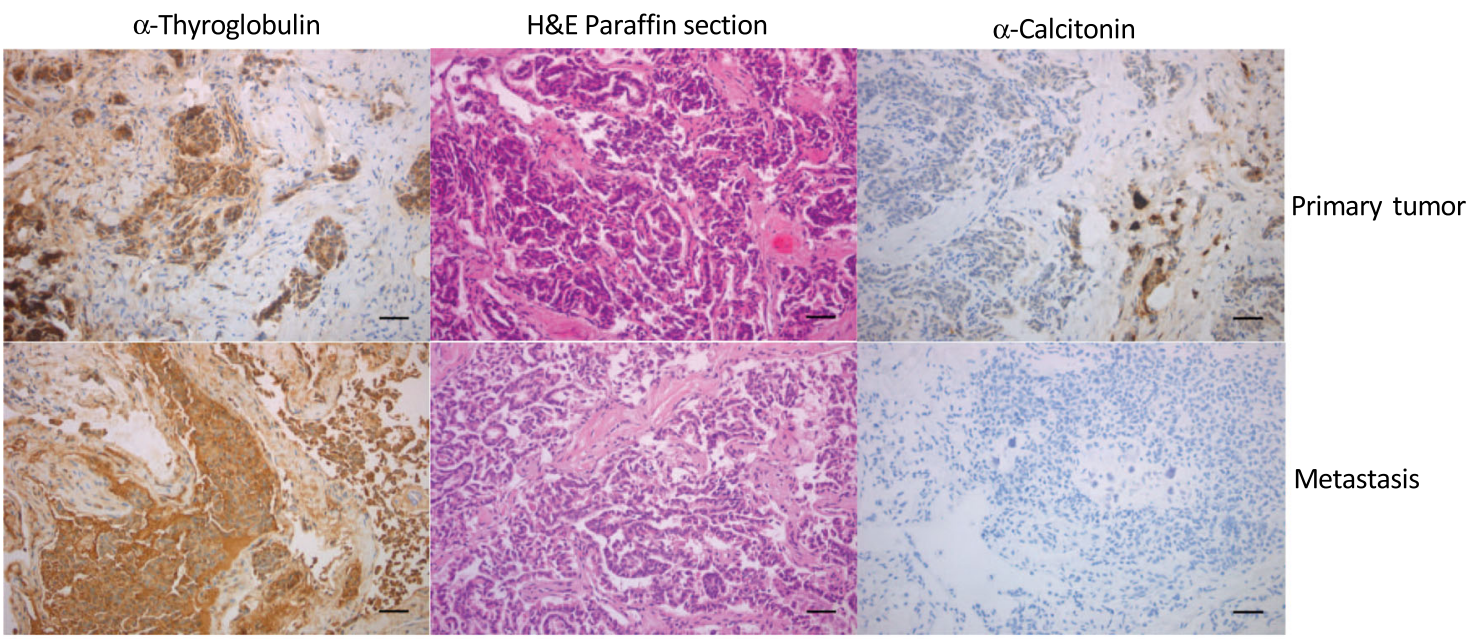

b

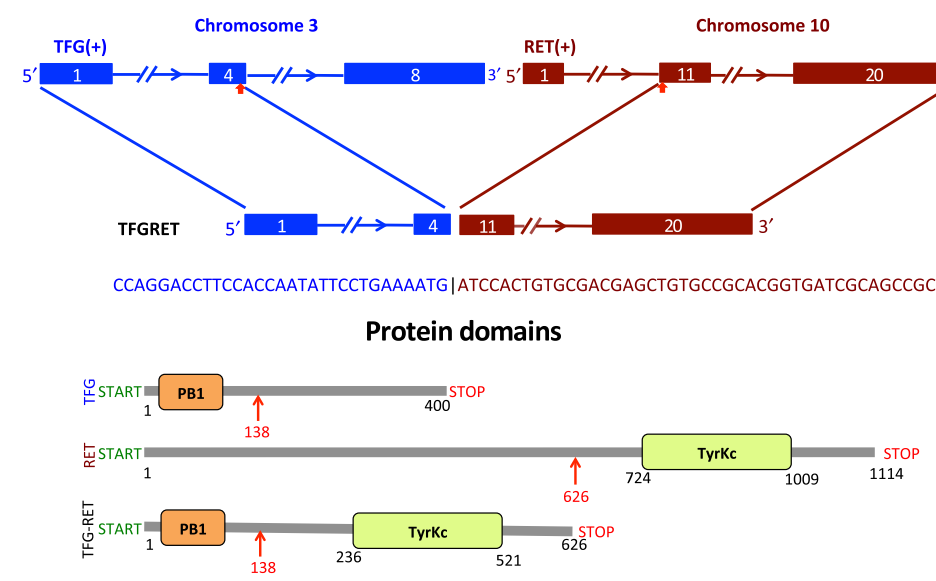

C

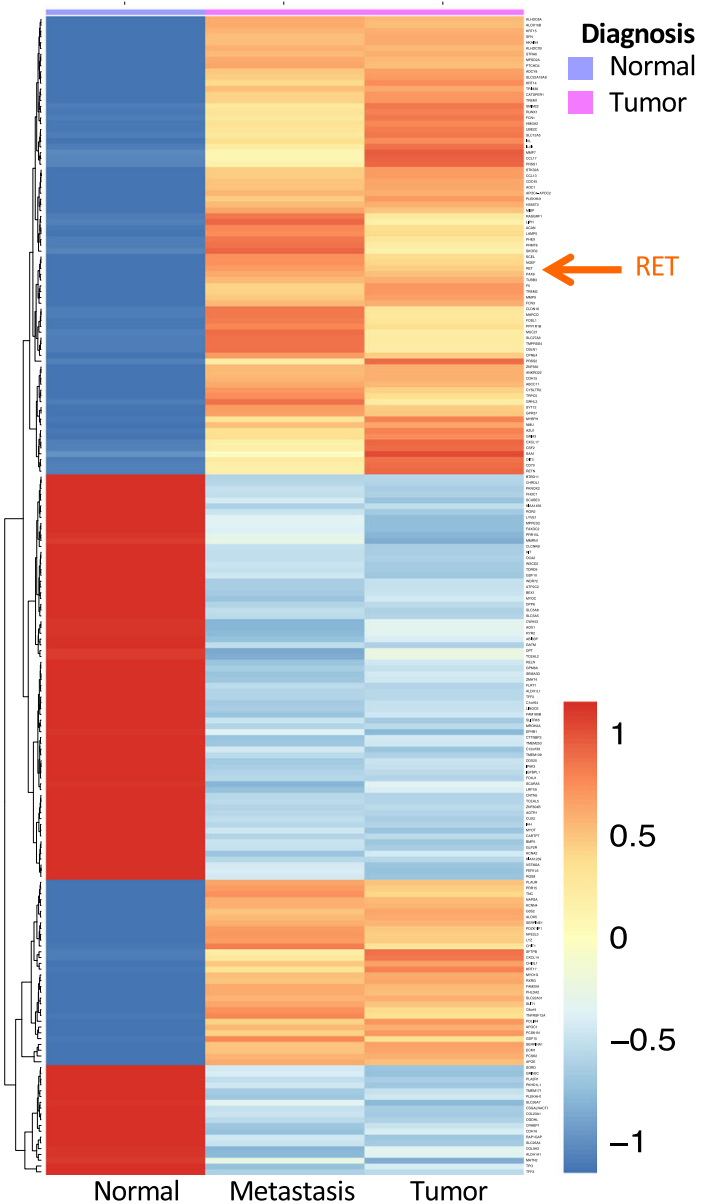

Fig. 1 A novel fusion product is identified in patient PTC sample. a Immunohistochemical analysis of patient samples. $\alpha$-thyroglobulin expression was detected in both primary and LN metastasis tissues, implying that the tumor is a PTC. Haemotoxylin and eosin (H\&E) staining shows follicular nature of the tumor. $\alpha$-calcitonin was detected in some cells of the primary tumor tissue implying c-cell hyperplasia and there was no $\alpha$-calcitonin expression in the metastatic tissue (magnification $\times 20$, Bar $50 \mu \mathrm{m}$ ). Presented are representative data from a diagnostic staining procedure. b Diagrammatic representation of TFG-RET protein. TFG-RET contains 626 amino acids, and is a fusion between the N-terminal part of TFG protein and C terminus of RET protein. The TFG region (1-138) contains a PB1 domain, whereas the RET region (139-626) contains the tyrosine kinase domain. $\mathbf{c}$ Heat map of the RNA-seq analysis of normal vs tumor vs metastasis as mentioned in the Methods section. RET overexpression is indicated in the tumor.

(Fig. 3a). PB1 domains are evolutionarily conserved proteinprotein interaction motifs contributing to formation of oligomers ${ }^{17}$. We hypothesized that the PB1 motif in the TFG-domain could possibly contribute to the formation of dimers and/or multimers of RET kinase, which is critical for the activation of the kinase. Immunoprecipitation of exogenously expressed RET constructs and the gene fusion revealed that TFG-RET readily forms dimers and multimers, which was confirmed by 
Table 1 Table listing the somatic mutations detected for the tumor and LN metastasis.

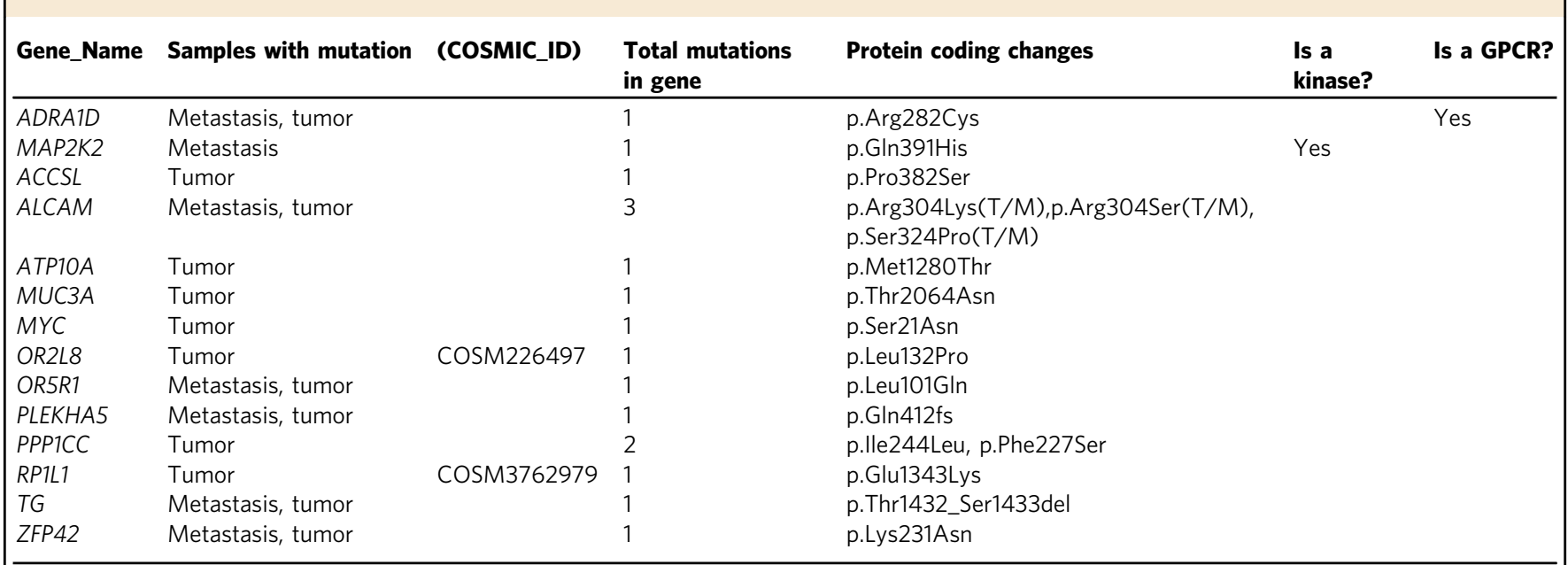

Enlists protein-altering gene mutations detected in exome analysis of tumor and LN metastatic lesions from the patients.

experiments employing crosslinking agents (Fig. 3b, c). As expected, the crosslinked wild-type kinase was not detected due to the limitation in the detection of larger oligomers with the employed gradient gels. Further, unlike the wild-type RET kinase, which is largely membrane-bound, a significant fraction of the TFG-RET fusion protein is detected in the cytosol of the cells stably expressing this gene fusion (Supplementary Fig. 3C, D). This could be attributed to the loss of membrane binding and other regulatory domains of full-length RET in the fusion, and is a characteristic of other RET fusions ${ }^{14}$.

PB1 domains have been demonstrated to undergo head-to-tail dimerization $^{18}$. DIX (dishevelled and axin) and PB1 domains are polymerizing domains that exhibit high structural similarities. We performed structural analysis of the TFG-PB1 domain by aligning it with the published structures of the DIX domain of Dvl2 and the PB1 domain of p62. We developed a TFG-PB1 model based on the DIX polymer of Dvl2, which displayed conserved residues and the coiled-coiled (CC) domain (Fig. 4a, b). We performed triple mutations (K14E, R22E and R23E) and deletion of the CC domain ( $\Delta 97-124)$ in TFG-RET. As expected, this lead to a reduced oligomerization of TFG-RET fusion as shown by gel filtration experiments (Fig. 4c-e). Further, mutations in the PB1 domain and in the CC domain led to reduced levels of phospho-RET (Y905), an autophosphorylation site of the RET kinase, which is also required for activity of RET kinase $^{19}$, as well as downregulation of other signaling pathways (Supplementary Fig. 4A-C). However, mutating three conserved residues in the PB1 domain but not in the CC domain compromised the stability of the protein (Supplementary Fig. 4D). We then performed soft agar colony formation experiments, which revealed that the integrity of the TFG-domain is not only required for TFG-mediated RET oligomerization but also for RET-mediated transformation (Fig. 4f). These data confirmed the critical role for TFG-domain-dependent oligomerization in RETmediated tumorigenesis.

Upregulation of ubiquitination-associated proteins in PTC. With an aim to further identify factors that are differentially expressed in both tumor and LN metastatic lesions of the same patient, we performed label-free quantitative proteomics as described in the Methods section (Supplementary Data 4). These data uncovered several factors that are specifically expressed in tumors and LN metastatic lesions (Fig. 5a, Supplementary Fig. 5A and Tables 2 and 3). We also observed that many of the factors that are upregulated on the mRNA levels are not detected on the protein levels, which suggest post-transcriptional regulation (Supplementary Data 5). Interestingly, we detected several members of the ubiquitin signaling machinery upregulated on the mRNA level in the tumor and LN metastatic lesion (Fig. 5 and Supplementary Fig. 5). We have extended the proteomic analysis further to 4 more patients and we consistently identified HUWE1 as one of the prime factors that are upregulated in the tumor and metastatic lesions (Fig. 5b, c, Supplementary Fig. 5C and Supplementary Data 6). We also detected STAT3 and KRAS being upregulated in the tumor and metastatic tissue of a subset of patients (Fig. 5c). As HUWE1 is more consistently detected in several patients, we focused further on the role of HUWE1 in mediating PTC tumorigenesis. HUWE1 is a HECT domaincontaining E3 ubiquitin ligase that regulates the stability of various cellular targets and has been shown to exhibit both tumor suppressor and oncogenic functions ${ }^{20,21}$. For instance, HUWE1 can target oncoproteins like N-MYC, C-MYC, MCL1 and p5322-25. In addition, deubiquitinases like USP9X and UBP7 are also highly expressed in both tumor and LN metastatic lesions of the patient with TFG-RET fusion-expressing tumor tissue (Supplementary Fig. 5A, B). In order to test our proteomics observations in a larger cohort of patient samples, we analyzed the expression of HUWE1, USP9X and USP7 in protein lysates isolated from fresh frozen PTC patient samples (7 normal, 8 tumor, 3 metastatic lesions). Our data show that expression of HUWE1, USP9X and USP7 are higher in the tumor and metastatic lesions of many patients, compared to matched normal tissue irrespective of their mutational status (Supplementary Fig. 5D, E). We also observed an upregulation of HUWE1, USP9X and USP7 in Nthy-TFG-RET cells (Fig. 5d, Supplementary Fig. 5F). Expression of HUWE1 was enhanced on the mRNA level as well in Nthy-TFG-RET expressing cells (Fig. 5e).

We next investigated whether these ubiquitin-associated proteins have any functional role in cells expressing TFG-RET. For this, we employed a transient knockdown approach employing siRNAs against HUWE1, USP9X and USP7. We validated the knockdown efficiency of the siRNAs (2 sets against each target were used, with each set consisting of 2 different siRNAs; Supplementary Fig. 6A). Although HUWE1 and USP7 siRNAs were quite effective, USP9X siRNAs only displayed a 50\% downregulation of the protein (Supplementary Fig. 6A). MTT 


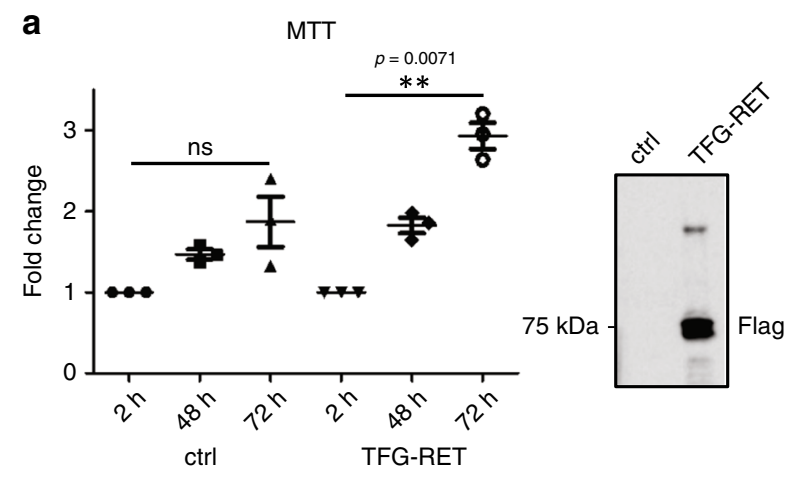

b EdU

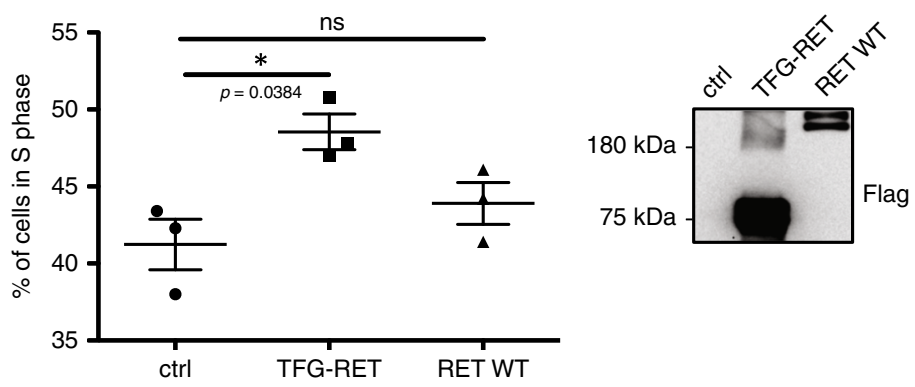

C

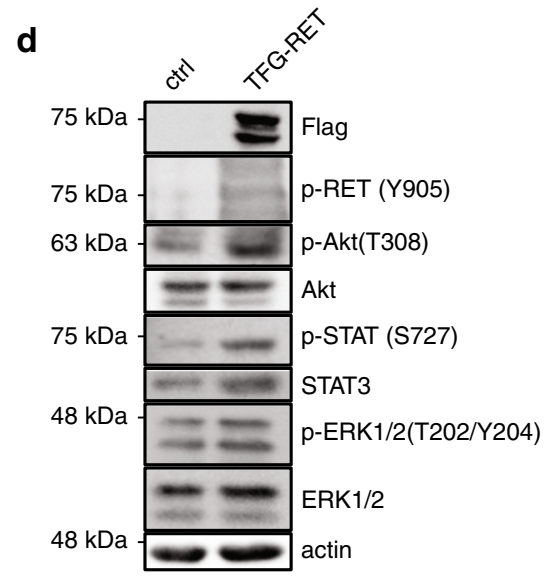

e
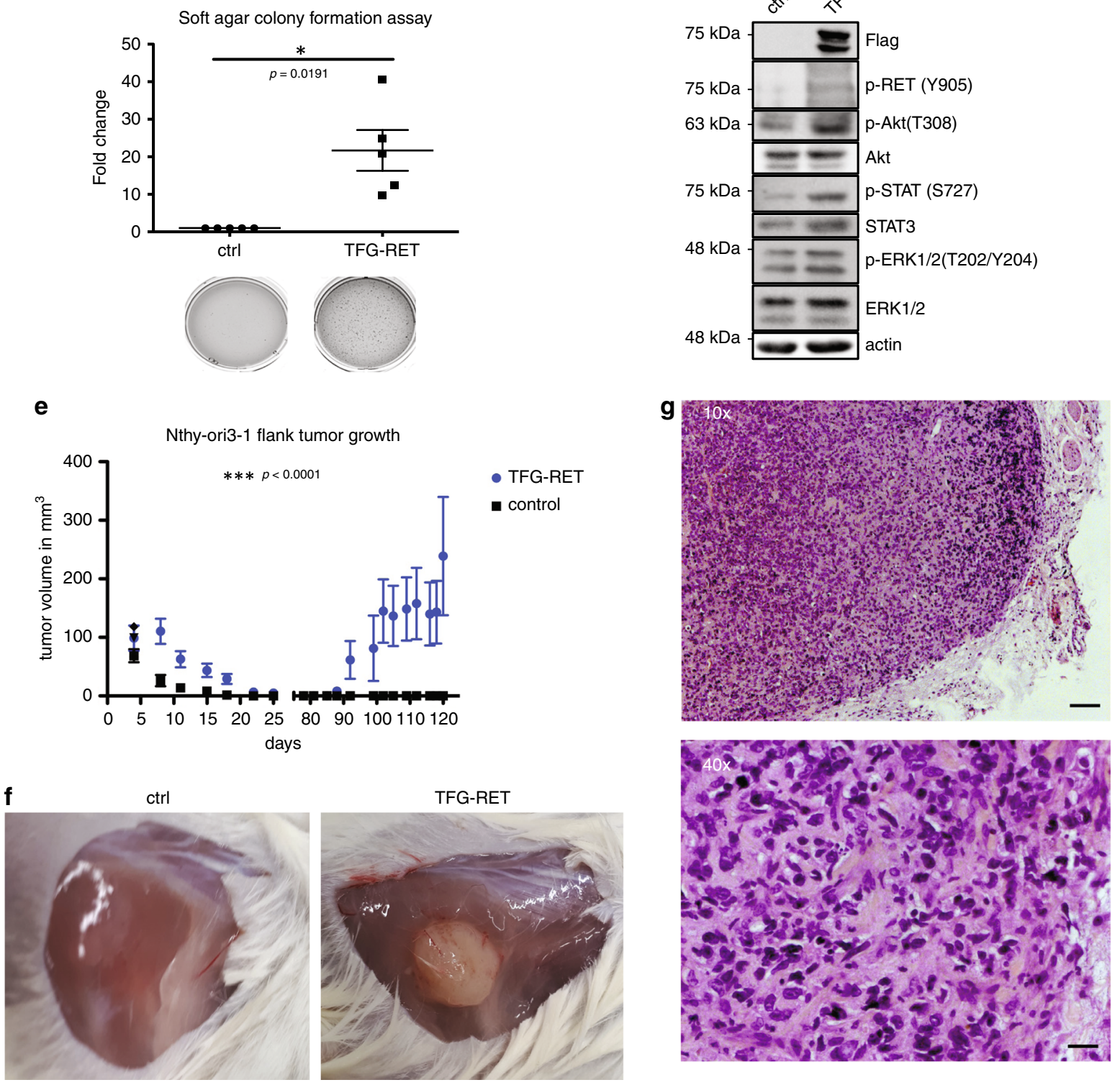

assays indicated that knockdown of HUWE1 and USP7 led to a significant reduction in the viability of Nthy-TFG-RET cells after 48 and $72 \mathrm{~h}$ (Fig. 6a and Supplementary Fig. 6B). In cellular proliferation experiments monitored by EdU incorporation and direct cell counting after 48 and $72 \mathrm{~h}$, it was observed that HUWE1 knockdown significantly reduced cell proliferation, although this was not always the case with USP9X and USP7 (Fig. 6a and Supplementary Fig. 6B, C). We hypothesized that HUWE1 and DUBs are probably required for RET-mediated transformation. Indeed, colony formation assays revealed a significant reduction in the transforming ability of Nthy-TFGRET cells with HUWE1 knockdown (Fig. 6b). USP7 knockdown 
Fig. 2 TFG-RET expression induces increase in cell viability, cell proliferation and oncogenic transformation in vivo. a Immortalized normal human primary thyroid follicular epithelial cells (Nthy-ori 3-1 cells) were infected with FLAG-tag expressing pPHAGE C-TAP TFG-RET virus particles and selected along with empty vector control. Cells were seeded in 96-well plates and subjected to MTT assay post 48 and $72 \mathrm{~h}$ as described in Methods. TFG-RET expression leads to an increase in cell viability after $72 \mathrm{~h}$ (absorbance relative to $0 \mathrm{~h}$ ). Error bars represent $\pm \mathrm{SEM}(n=3)$. ${ }^{\star \star} p<0.01$, paired $t$-test, twotailed distribution. Western blot confirmed TFG-RET expression. The fold increase in the absorbance (OD) was depicted as mentioned in the Methods section. $\mathbf{b}$ Nthy-TFG-RET cells along with empty vector control carrying cells were analyzed using EdU DNA synthesis assay as mentioned in the Methods section. Cells were seeded in $60 \mathrm{~mm}$-cell culture plates and analyzed for cell proliferation after $72 \mathrm{~h}$. Stable expression of TFG-RET leads to an increased cell proliferation. Error bars represent $\pm \operatorname{SEM}(n=3)$. ${ }^{\star} p<0.05$, paired $t$-test, two-tailed distribution. Stable expression of RET WT induced slight increase in cell proliferation, although this effect was not significant. The expression of wild type and fusion RET constructs were confirmed by western blots. Shown are the percentage of cells in the S phase for the analyzed samples. c Nthy-TFG-RET cells along with empty vector control were cultured in soft agar for 2 weeks followed by staining with crystal violet. Error bars represent $\pm \operatorname{SEM}(n=3)$. Paired $t$-test, two-tailed, three independent experiments: two with technical duplicates and one experiment with single technical replicate, $p$-value $<0.05$. Shown are the fold changes in the number of colonies between the control and TFG-RET expressing cells. d Western blots for indicated proteins ( $p$, phosphorylated) in lysates from Nthy-TFG-RET cells along with empty vector control. Expression of TFG-RET upregulated various cancer-associated signaling pathways. Shown are representative data from at least three independent experiments. e Growth of $4 \times 10^{6}$ subcutaneously injected thyroid Nthy-ori 3-1-cells in vivo. Nthy-TFG-RET cells formed solid, slowly progressing tumors in mice ( $n=8$ per group). For statistical analysis, two way ANOVA was performed. Error bars represent \pm SEM. ${ }^{\star \star \star} p<0.0001$. f Injection site of Nthy-ori 3-1 cells after 115 days of tumorigenesis. Nthy-TFG-RET injected mice developed solid tumors, whereas no tumors or neoplasia occurred in Nthy-ori 3-1 control injected mice $(n=8)$. $\mathbf{g}$ Histopathology of Nthy-TFG-RET tumors in H\&E sections. The larger overview demonstrates distinct tumors with surrounding healthy tissue (scale bar $=100 \mu \mathrm{m})$. Higher magnification shows malignant growth patterns, atypical cell morphology and size, abrogation of the cell polarity and abnormal nuclei (scale bar $=50 \mu \mathrm{m}$ ). Shown here are representative images obtained from evaluation of H\&E staining of multiple tumor tissue sections.

showed a tendency for reduced transformation, whereas USP9X did not show any such tendency at least in the time frame of the experiment (Supplementary Fig. 6C). This might be because of the lesser knockdown efficiency of the siRNAs employed against USP9X (Fig. 6a). We failed to obtain stable knockdown of these targets with shRNAs in Nthy-TFG-RET cells despite repeated attempts. Nevertheless, to further corroborate these observations, we employed small molecule inhibitors targeting HUWE1 (BI8622 and BI8626), DUBs (WP1130) and RET kinase activity (presented in Table 4). Treatment with these compounds significantly reduced RET-mediated colony formation (Fig. 6c). One of the suggested HUWE1 inhibitor (BI8622) almost completely inhibited the growth of TFG-RET transformed cells. We confirmed that both BI8622 and BI8626 inhibited HUWE1mediated ubiquitination in cells by performing ubiqapture experiments (Supplementary Fig. 6D). Further, we also detected that these inhibitors reduced the proliferation of Nthy-TFG-RET cells, probably with a G1-S blockade, as they did not strongly induce cell death (Supplementary Fig. 7A-C). These results suggest that targeting the ubiquitin signaling machinery might possibly be explored as a strategy to combat RET-mediated oncogenesis in thyroid cancers.

\section{Discussion}

The discovery of oncogenic gene fusions including BCR-ABL and ALK fusions has led to the development of successful targeted therapies, particularly in hematologic malignancies ${ }^{26}$. With the recent advances in next-generation sequencing techniques, there has been a massive increase in the number of molecular fusions described, especially in solid tumors. A TCGA study that aimed at the genomic characterization of 496 PTCs illustrated the mutual exclusivity of genetic driver alterations in PTCs, which further emphasizes the importance of precision medicine in the treatment of cancer ${ }^{15}$. PTC contributes to almost $80 \%$ of thyroid cancers and RET fusions are the most commonly detected gene fusions in PTCs ${ }^{15}$. More than 20 RET fusions have been reported in PTCs, of which the most common are the RET/PTC1 and RET/PTC3 fusions ${ }^{27-29}$. Recent studies have also identified RET fusions in lung and colon adenocarcinoma, breast cancer as well in MTCs, a more aggressive form of thyroid cancer $^{30,31}$. By performing genomic analysis of a single PTC patient, we identified a novel, oncogenic RET fusion in both tumor and LN metastatic lesions, whose expression readily transformed immortalized human thyroid cells and induced tumor formation in mice.

The PB1 domain in the TFG fragment has a key role in regulating the oligomerization and activation of the RET kinase. PB1 domains are capable of forming head-to-tail oligomers, which is a key step in signalosome assembly ${ }^{18}$ and hence have an important role in the regulation of protein signaling. All DIX-like domains, including the PB1 domain have lower $K_{\mathrm{d}}$ values, suggesting that they will produce oligomers in solution ${ }^{18}$. Mutation analysis showed that the PB1 domain-mediated oligomerization contributed to the autophosphorylation and/or activation of the RET kinase. Previous studies have shown that auto- and crossphosphorylation at Tyr905 is required for RET kinase activity and mutation of this residue with others contributed to reduced RET activity $^{32}$. Consistent with these observations, we detected that mutations in the oligomerization interface of the TFG-domain reduced Tyr905 phosphorylation in the TFG-RET fusion (Supplementary Fig. 4). Further, these mutations impaired RETmediated oncogenic transformation (Fig. 4F). Together, this suggests that targeting the TFG-domain could possibly be a strategy to combat TFG-RET-mediated tumorigenesis, given the fact that most of the kinase inhibitors exhibit off target effects. Further guided-fusion detection studies in large cohorts of PTC patients are required to evaluate the frequency of this gene fusion. We also detected thus far unreported mutations in the coding regions of other interesting targets like the MAPK2K2 and several upregulated factors whose significance in driving PTC tumorigenesis needs further studies. Similar to the observation in the TCGA studies, we also detect that the frequency of the proteinaltering gene mutations are in general low in PTCs (Supplementary Fig. 1C) ${ }^{15}$.

By employing proteomics, we further demonstrated that several members of the ubiquitin signaling machinery are deregulated in both tumor and LN metastatic lesions and interestingly, we detected high HUWE1 expression in RET transformed cells. HUWE1 was also identified in patients without known RET fusions and further studies are clearly warranted (Fig. 5b). In addition to the kinome, the ubiquitinome has recently emerged as a favorable druggable target as there are several clinical trials going on with drugs targeting the ubiquitin signaling machinery $^{33}$. Here, we present evidence that targeting HUWE1 or DUBs 


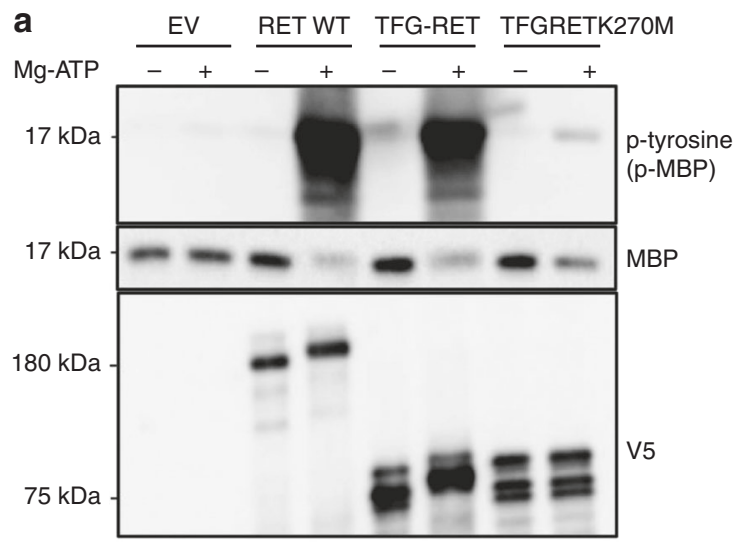

C

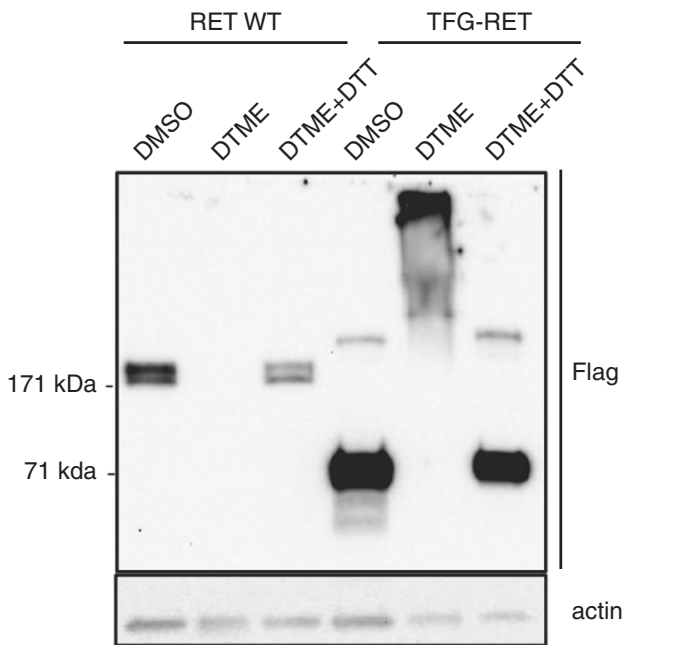

b

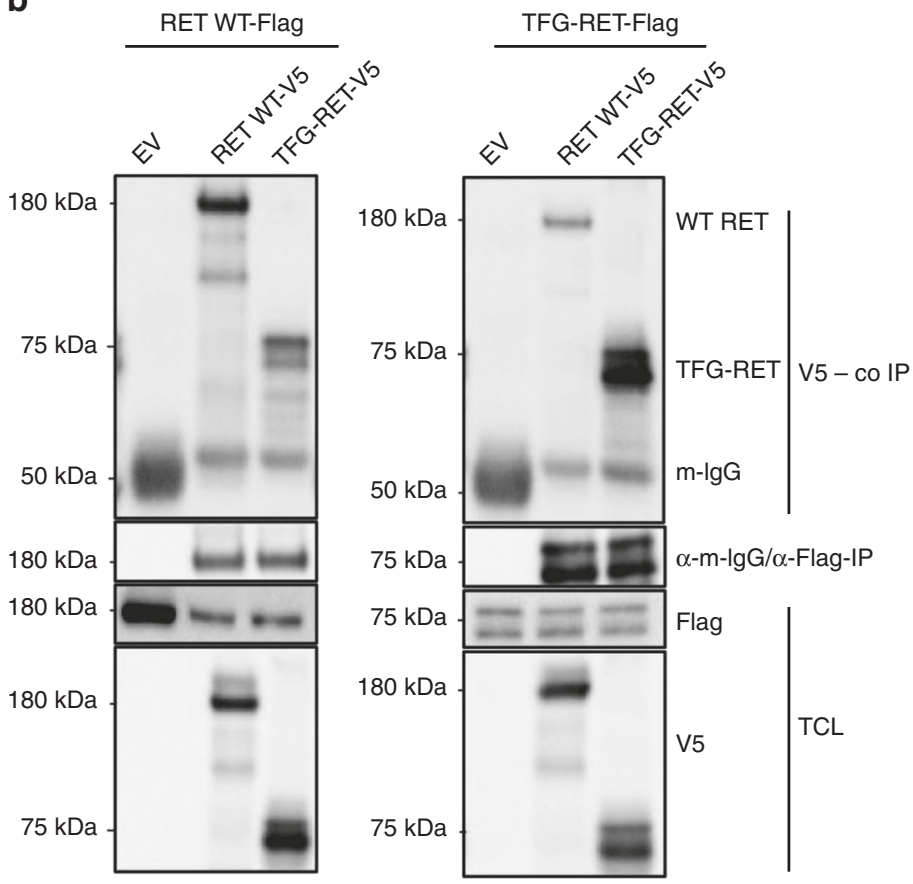

Fig. 3 TFG-RET exhibits kinase activity and forms oligomeric complexes. a HeLa cells were transiently transfected with V5-tagged wild-type RET, TFGRET and kinase dead TFG-RET K270M along with empty vector. After $48 \mathrm{~h}$ cells were lysed and subjected to immunoprecipitation using anti-V5 antibody. After subsequent washes, the immunoprecipitated proteins were used for in vitro kinase assay, as described in Methods. TFG-RET phosphorylated MBP, thus exhibiting kinase activity like wild-type RET kinase. This kinase activity was abrogated in the TFG-RET kinase dead mutant ( $p$, phosphorylated). Shown are representative data from at least three independent experiments. b HeLa cells were co-transfected with FLAG-tagged and V5-tagged wild-type RET and TFG-RET as indicated, followed by anti-FLAG immunoprecipitation after $48 \mathrm{~h}$. Lysates were analyzed by western blotting (TCL, total cell lysate). Wild-type RET (FLAG ${ }^{\circledR}$ ) co-immunoprecipitated with wild-type RET (V5) and also with TFG-RET (V5) (left panel), similarly, TFG-RET (FLAG ${ }^{\circledR}$ ) also co-

immunoprecipitated with both wild-type RET (V5) and TFG-RET (V5). Empty vector controls were included. Shown are representative data from at least three independent experiments. c Nthy-ori 3-1 cells stably expressing wild-type RET and TFG-RET were treated with DTME and DTME + DTT. Upon chemical crosslinking with DTME, similar to wild-type RET, TFG-RET also formed high molecular weight heteromeric complexes and these complexes were disrupted upon reduction with DTT (lane 3 and 6). Shown are representative data from at least two independent experiments.

like USP9X and UBP7 may be an attractive strategy to combat RET-mediated oncogenesis. The possible role of these ubiquitination-associated proteins in PTCs was further highlighted by our data from a larger cohort of PTC patient samples where these proteins seem to be overexpressed (Supplementary Fig. 5D, E). Whether these targets are also expressed in PTCs with other common mutations like BRAF, NRAS and NTRK1 fusions requires further analysis. It would also be very interesting to check whether upregulation of these proteins is correlative to RET fusions in general, not just limited to PTCs.

The determination of the malignancy status of thyroid nodules and accurate stratification of thyroid lesions are still challenging in the management of thyroid cancer. In a recent analysis of 56 patients suffering from PTC it was shown that RET rearrangements were associated with a higher risk of developing LN metastasis and an elevated risk of developing iodine refraction ${ }^{34}$. Improved molecular characterization of needle biopsies would ease stratification of thyroid nodules and would furthermore guide the development of "personalized therapeutics" for PTC patients who are refractory to conventional RAI treatment modalities. Many of these targets described here could potentially be pursued for molecular diagnosis as well as patient stratification. Overall, our studies, in addition to unveiling an oncogenic gene fusion, have identified druggable targets, which open further avenues of treating PTCs, the most common type of thyroid malignancies.

\section{Methods}

Patient sample acquisition. Informed consent was obtained from the patients prior to surgery. We have the ethical approval for the study granted by the "Landesärztekammer", which is also the institutional approval (Study number: 837.119.15 (9888)). Tumor and normal tissue were harvested intraoperatively from consented patients following standard operative procedures. For patient \#1, the right thyroid lobe comprised mostly of tumor, while the left thyroid lobe predominantly was normal tissue. Ultrasound examination revealed that the patient had multiple lateral lymph node metastases. The staging was: pT4b $(5.3 \mathrm{~cm}$ tumor $)$, pN1b (17 metastases in 29 lymph nodes), M1 (pulmonary metastases), multifocal bilateral tumor, some with follicular pattern and also in the left lobe, capsular invasion but no invasion of adjacent structures. Routine Sanger sequencing was performed to monitor the mutational status of BRAF wild type and $\mathrm{K}, \mathrm{H}$, NRAS. 

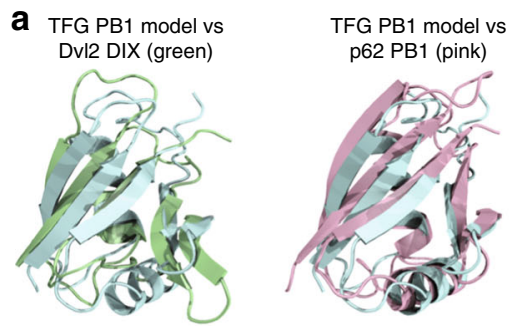

hDvl2 polymer 4WIP

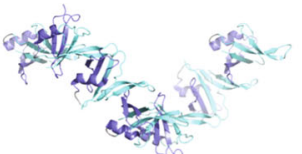

TFG PB1 polymer MODEL
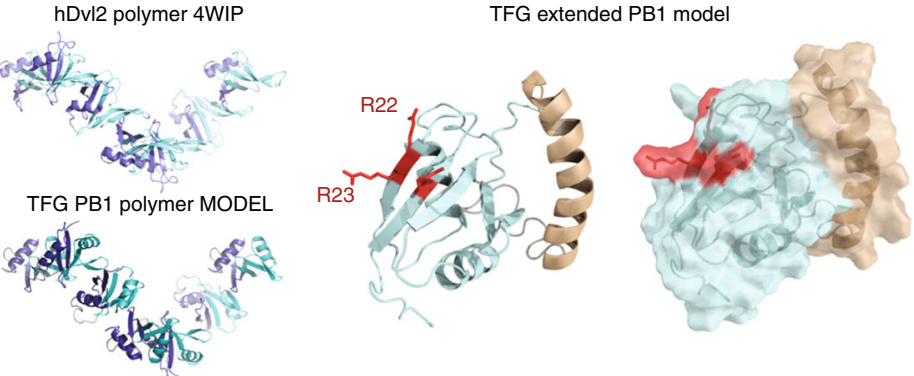

b
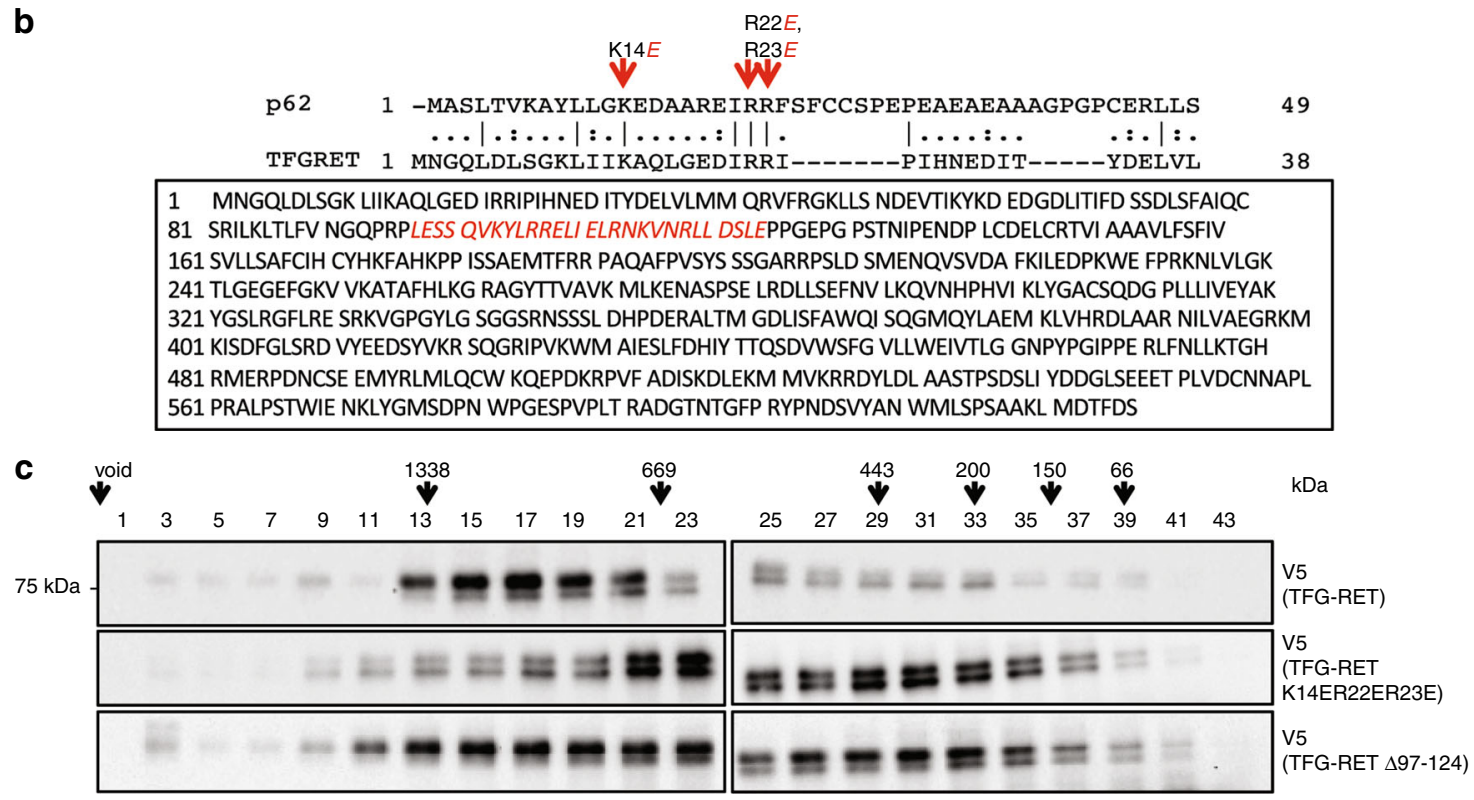

d

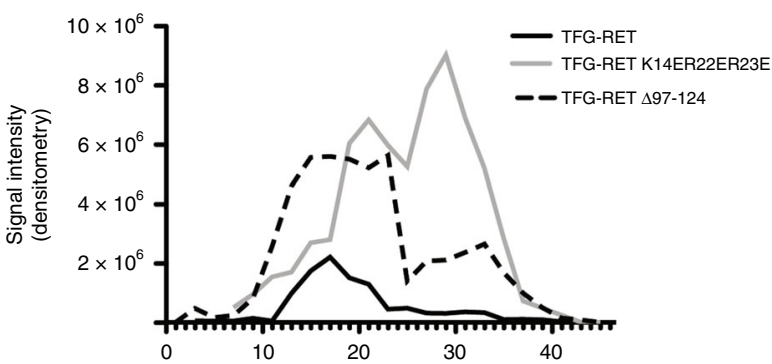

e

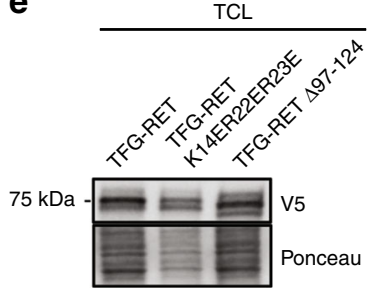

f

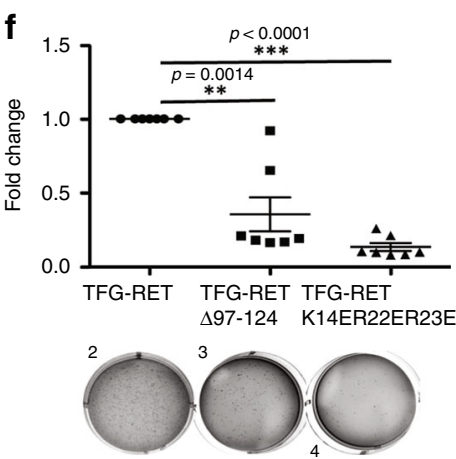

g

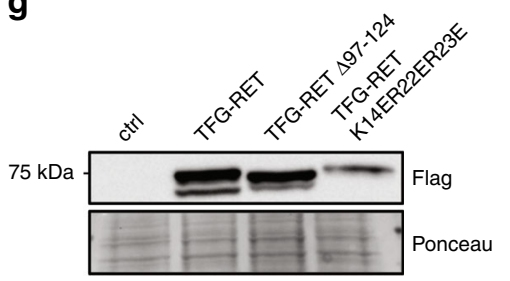

Normal, tumor and metastasic (from lymph node) tissue were taken for further analysis. A procedure involving a total thyroidectomy with therapeutic central and right lateral lymph node dissection (neck dissection, levels II-VII) was performed. The lymph node was chosen because the macroscopic appearance was clearly metastatic. Histology described a multifocal, bilateral follicular differentiated papillary thyroid carcinoma (pT4b, pN1, M1 (lung), Stage II, Classification according to 1997). All other tissue samples were provided by the tissue bank of the
University Medical Center Mainz in accordance with the regulations of the tissue bank.

Isolation of protein, DNA and RNA from patient tissue. The tumor tissue was incubated in collagenase- $\mathrm{D}$ solution $(1 \mathrm{mg} / \mathrm{mL})(11088866001$, Roche $)$ at $37^{\circ} \mathrm{C}$ for $2 \mathrm{~h}$ and then transferred to a 6-well plate and cut into smaller pieces with a scalpel 
Fig. 4 TFG domains have a role in the TFG-RET heteromerization and oncogenicity. a Structural comparison of TFG-PB1 vs DVI2 DIX or p62 PB1 and the TFG-PB1 model showing the head region (deep blue) and tail region (teal) based on the DIX polymer of hDvl24WIP (head (slate), tail (aquamarine)). b Mutations in PB1 domain (top panel)-Comparison of PB1 sequences (not entire) of p62 and TFG-RET. Conserved residues Lys14, Arg22 and Arg23 were substituted with oppositely charged Glu to create PB1 domain mutant TFG-RET (TFG-RETK14ER22ER23E). CC domain (bottom panel)-Leu97 to Glu124 comprise the CC domain of TFG-RET. This domain was deleted to obtain the CC deletion mutant of TFG-RET (TFG-RET $\Delta 97-124)$. c, $\mathbf{d}$ Lysates collected from HeLa cells transiently expressing indicated constructs were loaded on Superose- 6 gel filtration column. Collected fractions (every second fraction) were subjected to western blotting. TFG-RET, TFG-RETK14ER22ER23E as well as TFG-RET $\Delta 97-124$ were detected using V5 tag. Most of TFG-RET was detected in high molecular weight fractions, while TFG-RETK14ER22ER23E shifted toward the lower molecular weight fractions. (Molecular weight corresponding to the elution volume are indicated by the arrows above). Shown are representative data from at least two independent experiments. e Protein expression of TFG-RET, TFG-RETK14ER22ER23E and TFG-RET $\Delta 97-124$ (pcDNA DEST V5. His vector) following transient transfection in HeLa cells was verified by Western blot analysis of the cell lysates (cytosolic fraction obtained after ultracentrifugation). Shown are representative data from at least two independent experiments. $\mathbf{f}$ Soft agar colony formation assay. Nthy-ori 3-1 cells stably expressing indicated constructs were cultured in soft agar for 2 weeks followed by staining with crystal violet. Error bars represent \pm SEM $(n=3)$. Paired $t$-test, two-tailed. Three independent experiments were performed: one experiment with technical triplicates, and two experiments with technical duplicates. ${ }^{\star \star \star} p$-value $<0.0001,{ }^{\star \star} p$-value $<0.05$. Shown are the fold changes in the number of colonies between the analyzed samples. $\mathbf{g}$ Immunoblot analysis of expression of TFG-RET, TFG-RET $\Delta 97-124$ and TFGRETK14ER22ER23E in Nthy-ori 3-1 cells. Shown are representative western blots from the experiments presented in $\mathbf{f}$.

and ground using the thumb press of a syringe plunger. The suspension was passed through a $70-\mu \mathrm{m}$ cell sieve, centrifuged at $240 \times g$ for 5 min and subsequently washed with cold PBS. The cell pellet was re-suspended in lysing buffer $(250 \mathrm{mM}$ $\mathrm{NaCl}, 50 \mathrm{mM}$ Tris-HCl pH 7.5, $10 \%$ glycerol, $1 \%$ Triton X-100, $0.2 \mathrm{mM} \mathrm{Na}_{3} \mathrm{VO}_{4}$ and protease inhibitor cocktail (539131, Calbiochem)) and kept on ice for $10 \mathrm{~min}$. The cell suspension was sonicated (three times $10 \mathrm{~s}, 50 \%$ amplitude) and centrifuged at $17,000 \times g, 4^{\circ} \mathrm{C}$ for $10 \mathrm{~min}$. Then, the supernatant was used for mass spectrometric analysis. DNA and RNA were isolated using AllPrep DNA/RNA Mini Kit (80204, Qiagen) following the manufacturer's protocol.

For western blot analysis of proteins from patients' tissue, samples-normal, tumor, metastasis-were lysed in RIPA buffer ( $25 \mathrm{mM}$ Tris- $\mathrm{Hcl}$ (pH 8.0), $1 \mathrm{mM}$ EDTA SDS, $1 \%$ Triton X-100, 0.1\% Sodium deoxycholate, $0.1 \%$ SDS and $150 \mathrm{mM}$ $\mathrm{NaCl}$ ), sonicated and centrifuged. Supernatants were mixed with Laemmli buffer, boiled and subsequently analyzed by western blotting.

Sequencing. RNA-seq libraries were prepared using TruSeq RNA Sample Preparation v2 kit (RS-122-2001, Illumina). Exome capture was performed using the Agilent SureSelect Human All Exome kit (50 Mb; G3370L, Agilent). RNA-seq and Exome capture libraries were sequenced on HiSeq (Illumina) to generate $2 \times 150 \mathrm{bp}$ and $2 \times 75$ bp paired-end data, respectively.

Exome data analysis. Burrows-Wheeler Alignment (BWA) software set to default parameters was used to map sequencing short reads to UCSC human genome (GRCh37) using local realignment, duplicate removal and raw variant calling ${ }^{35,36}$. Strelka was used for somatic variant calling on tumor and its matched normal BAM file ${ }^{37}$. Known germline variants represented in the Exome Aggregation Consortium (ExAC) were filtered out ${ }^{38}$.

RNA-seq data analysis. GSNAP (Genomic Short-read Nucleotide Alignment Program) was used to align RNA-seq reads to the human genome version NCBI GRCh $37^{39}$. Differential gene expression analysis was performed using DESeq2 ${ }^{40}$. Fusions were identified using a computational pipeline called GSTRUCT-fusions ${ }^{41}$.

CDNA isolation and PCR. cDNA was synthesized from total RNA isolated from primary tumor of patient using RevertAid reverse transcriptase cDNA Synthesis Kit (EP0441, Thermo Fisher Scientific) according to the manufacturer's protocol. The cDNA was analyzed for the presence of TFG-RET fusion transcript by PCR amplification using primers that amplify the fusion region. PCR amplification of cDNA (diluted 1:10 in water) was carried out using Q5 High-Fidelity DNA polymerase (New England Biolabs, M0491L), in the presence of $10 \mathrm{mM}$ DNTPs, 10 $\mu \mathrm{M}$ primers in $25 \mu \mathrm{L}$ reactions. PCR reaction was set up as described below in a thermo cycler:

Denaturation: $98^{\circ} \mathrm{C}-30 \mathrm{~s}$

Amplification (35 cycles): $98^{\circ} \mathrm{C}-10 \mathrm{~s}, 69^{\circ} \mathrm{C}-30 \mathrm{~s}, 72^{\circ} \mathrm{C}-15 \mathrm{~s}$

Extension: $72^{\circ} \mathrm{C}-2 \mathrm{~min}$

Primer sequences:

TFG-RET 267 fwd-tgttaatggccagccaagac

TFG-RET 267 rev-taccacaagtttgcccacaa

TFG-RET 430 fwd-attagtgctaatgatgcaacgagt

TFG-RET 430 rev-cttgtgggcaaacttgtgg

The PCR products were subsequently subjected to gel electrophoresis in a $1 \%$ agarose gel and visualized under UV. For sequencing, the $430 \mathrm{bp}$ PCR product was purified using QIAquick PCR purification kit (Qiagen, 28104) according to the manufacturer's protocol and sequenced using TFG-RET 430 fwd primer.
Immunohistochemistry. H\&E staining was performed on formalin fixed paraffin embedded sections using standard laboratory procedures. Immunohistochemistry was performed on paraffin sections by using the DAKO-EnVision FLEX-kit (Dako, Glostrup, Denmark). Staining was performed on an immunostainer (Autostainer; Dako, Glostrup, Denmark) according to the manufacturer's instructions.

Plasmids and constructs. Wild-type RET and a kinase dead version of TFG-RET (TFG-RETK270M) were cloned into Entry vectors for subsequent gateway cloning into target vectors. pENTR221 TFG-RET, pENTR221 TFG-RETK14ER21ER22E and pENTR221 TFG-RET $497-124$ were synthesized from Thermo Fisher Scientific and cloned into expression plasmids pPHAGE C-TAP (FLAG and HA tagged), a kind from Prof. Dr. Christian Behrends, and Gateway ${ }^{\mathrm{max}}$ pcDNA $^{\mathrm{m}}$-DEST40 Vector (His and V5-tagged) (12274015, Thermo Fisher Scientific).

Cell culture. Nthy-ori 3-1 cells (90011609, Sigma) were cultured in RPMI-1640 medium supplemented with $10 \%$ heat inactivated $\mathrm{FBS}$ at $37^{\circ} \mathrm{C}$ in $5 \% \mathrm{CO}_{2}$. HeLa (DSMZ) and 293T cells (a kind gift from Dr. Andreas Ernst) were cultured in DMEM supplemented with $10 \%$ heat inactivated FBS at $37^{\circ} \mathrm{C}$ in $5 \% \mathrm{CO}_{2}$. For transient transfections, $5 \mu \mathrm{g}$ plasmid and $27 \mu \mathrm{L}$ of $10 \mathrm{mM}$ polyethylenimine (PEI) were mixed in $500 \mu \mathrm{L}$ of PBS and incubated for 15 min at room temperature. After incubation, transfection reagent was added dropwise to cells cultured in $100 \mathrm{~mm}$ plates. In order to generate Nthy-ori 3-1 cells empty vector/TFG-RET cell lines, we first transfected HEK293T cells with pPHAGE C-TAP, pPHAGE_CMV_C_FLAG_HA_IRES_Puro together with the pLenti package (HDM-VSV-G; HDMtatlb; HDM-Hgprn2 (gag-pol); RC-CMV-Rev1b) for lentiviral particle production. After $48 \mathrm{~h}$, the media containing the virus were sterile filtered and then added to Nthy-ori 3-1 cells in the presence of $8 \mu \mathrm{g} / \mathrm{mL}$ polybrene. After $24 \mathrm{~h}$, cells were selected with $2.5 \mu \mathrm{g} / \mathrm{mL}$ puromycin and the surviving pool of cells was expanded and maintained in puromycin $(2.5 \mu \mathrm{g} / \mathrm{mL})$ containing media.

Quantitative RT-PCR. Total RNA was extracted using TRIzol ${ }^{\mathrm{TM}}$ reagent (15596018, Thermo Fisher Scientific) according to the manufacturer's protocol Equal amounts of total RNA were used to synthetize the corresponding cDNA using RevertAid reverse transcriptase cDNA Synthesis Kit (EP0441, Thermo Fisher Scientific). To quantify gene expression levels, SYBR-Green (A25780, Thermo Fisher Scientific) based qRT-PCR was performed using the StepOnePlus ${ }^{\mathrm{mt}}$ RealTime PCR System (4376600). The expression level of HUWE1 normalized to expression of reference gene (18S or RSP13) was determined in triplicates.

Primer sequences. q-PCR primers:

HUWE1: Ref: NM_031407, for-TTGGACCGCTTCGATGGAATA, revTGAAGTTCAACACAGCCAAGAG

18s: Ref: NT 167214.1, for-AGAAACGGCTACCACATCCA, revCACCAGACTTGCCCTCCA

RPS13: Ref: NM 001017, for-CGAAAGCATCTTGAGAGGAACA, revTCGAGCCAAACGGTGAATC

SDS-PAGE and western blot. For SDS-PAGE, cell lysates with equal amounts of total proteins were prepared in SDS-sample buffer $(0.125 \mathrm{M}$ Tris-HCl, $\mathrm{pH} 6.8,4 \%$ SDS, $10 \%$ glycerol, $10 \mathrm{mM}$ DTT and bromophenol blue) followed by boiling at $95^{\circ}$ C for 10 min. Cell lysates were then loaded onto $4-15 \%$ mini-PROTEAN ${ }^{\oplus}$ TGX $^{\mathrm{m}}$ Precast Protein Gels (4561084, Bio-Rad) or 7.5\% polyacrylamide gels. The proteins were then transferred to nitrocellulose blotting membranes (10600001, GE Healthcare) by western blotting. For immunoblot analysis, membranes were blocked with $5 \%$ low-fat milk in phosphate-buffered saline (PBS) for $30 \mathrm{~min}$ at 

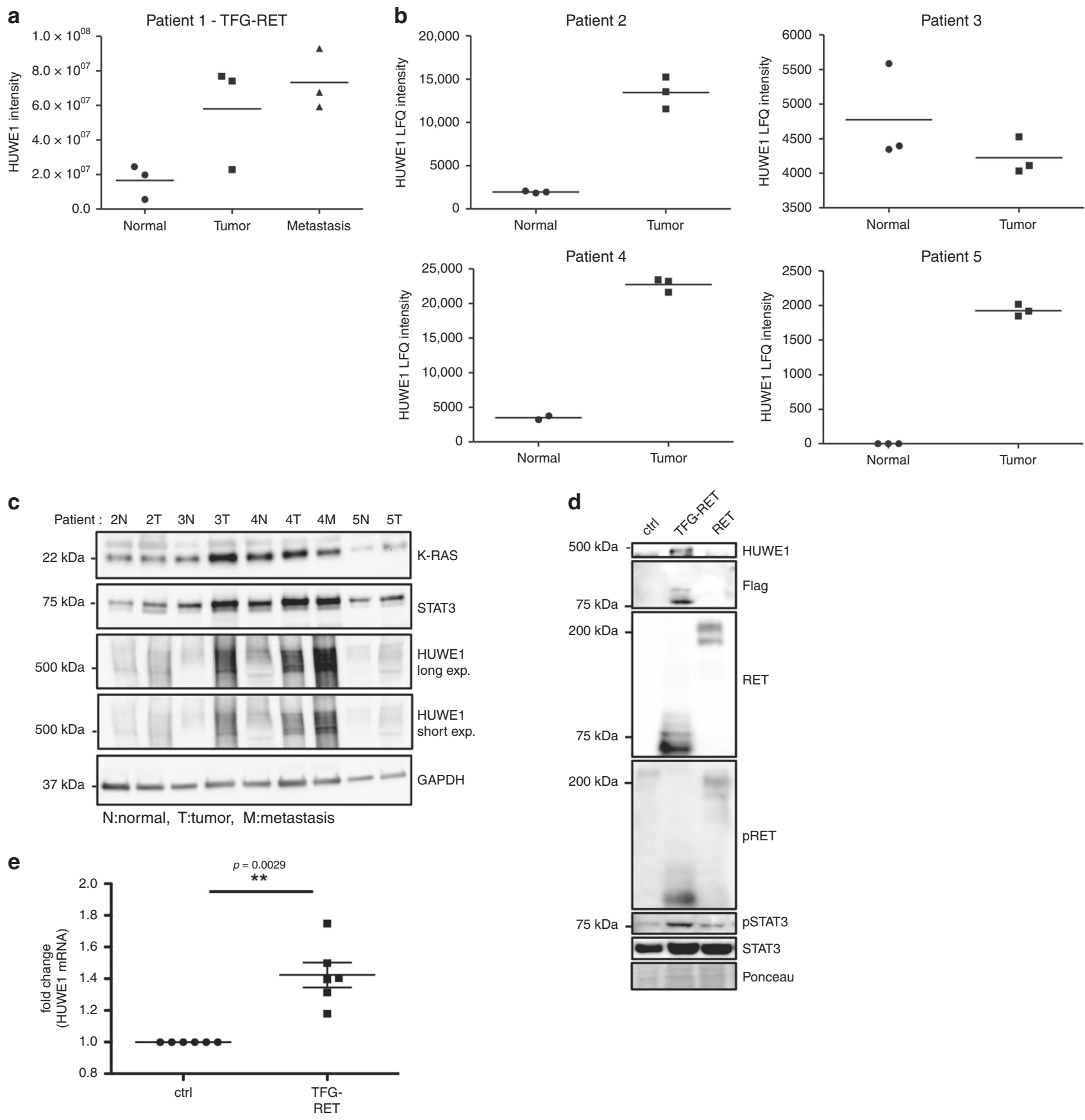

Fig. 5 Ubiquitin pathway-associated proteins are upregulated in PTCs. a Intensity values of HUWE1 from mass spectrometric data for patient 1. Shown are data from three technical runs from the same patient sample. b Intensity values of HUWE1 from mass spectrometric data for patients $2-5$. Shown are data from three technical runs from the same patient sample. c Western blots showing expression of selected proteins (KRAS, STAT3, HUWE1) from patients $2-5 . \mathrm{N}=$ normal, $\mathrm{T}=$ tumor and $\mathrm{M}=$ metastasis. $\mathrm{GAPDH}$ was employed as a loading control. Shown are representative western blots from at least two independent experiments. d Western blots for indicated proteins ( $p$, phosphorylated) in lysates from Nthy-ori 3-1 stably expressing FLAG-tagged (pPHAGE C-TAP vector) TFG-RET and RET. Cells transfected with empty vector served as control. Three independent experiments were performed ( $n=$ 3). e HUWE1 mRNA is overexpressed in Nthy-TFG-RET cells. Relative mRNA levels of HUWE1 were determined by real-time PCR. Error bars represent \pm SEM $(n=3)$. Three independent experiments each with technical duplicates were performed. Paired $t$-test, two-tailed, $p$-value $<0.05$.

room temperature and then incubated with primary antibodies in $3 \%$ bovine serum albumin (BSA; A7906, Sigma) overnight at $4^{\circ} \mathrm{C}$. Subsequently, the membranes were washed 3 times in PBS-T and incubated with horseradish peroxidase-coupled secondary antibodies for $1 \mathrm{~h}$ at room temperature, followed by washes as previously stated. The antigen-antibody complexes were detected by enhanced chemiluminescence (Immobilon Western Chemiluminescent HRP Substrate, WBKLS0500, Millipore) using Bio-Rad ChemiDoc ${ }^{\mathrm{TT}}$ Touch Imaging System (Bio-Rad). Quantification of Western blots was performed either by densitometry using the quantification software provided by Bio-Rad or by using Image J software (Open source image processing software http://imagej.net, Version: 2.0.0-rc-69/1.52i).
Antibodies. In this study, the following antibodies were used: Anti-thyroglobulin (M0781, Dako), anti-calcitonin (A0576, Dako) anti-phospho-tyrosine (P-Tyr1000) MultiMab ${ }^{\text {TM }}$ (8954S, CST), anti-Myelin Basic Protein (MBP) $(13344$, CST), anti-V5 antibody (Thermo Fisher Scientific, R960-25), anti-FLAG ${ }^{\circledR}$ M2-Peroxidase (A8592, Sigma), anti-phospho-RET (Y905) (3221S, CST), anti-RET (C31B4) (3223S, CST), anti-phospho-STAT3 (Ser727) (9134P, CST), anti-STAT3 (9139P, CST), anti-phospho-Akt (Thr308) (9275, CST), anti-Akt (C67E7) (4691, CST), anti-phospho-MEK1/2 (9154, CST), anti-MEK1 antibody (2352, CST), antiphospho-p44/42 MAPK (Thr202/Tyr204) (ERK1/2) (9101L, CST), anti-p44/42 MAPK (ERK1/2) (9102, CST), anti-M2-PK (S-1, Schebo Biotech), anti-histone H3 
Table 2 Enlists the proteins identified only in tumor patient sample through mass spectrometric studies.

Protein list-tumor only

\begin{tabular}{|c|c|c|}
\hline Accession & Entry & Description \\
\hline P01258 & CALC_HUMAN & Calcitonin OS $=$ Homo sapiens $\mathrm{GN}=\mathrm{CALCA} \mathrm{PE}=1 \mathrm{SV}=2$ \\
\hline P22748 & CAH4_HUMAN & Carbonic anhydrase $4 \mathrm{OS}=$ Homo sapiens $\mathrm{GN}=\mathrm{CA} 4 \mathrm{PE}=1 \mathrm{SV}=2$ \\
\hline P16671 & CD36_HUMAN & Platelet glycoprotein $4 \mathrm{OS}=$ Homo sapiens $\mathrm{GN}=\mathrm{CD} 36 \mathrm{PE}=1 \mathrm{SV}=2$ \\
\hline P35908 & K22E_HUMAN & Keratin type II cytoskeletal 2 epidermal $\mathrm{OS}=$ Homo sapiens $\mathrm{GN}=\mathrm{KRT} 2 \mathrm{PE}=1 \mathrm{SV}=2$ \\
\hline Q16647 & PTGIS_HUMAN & Prostacyclin synthase OS $=$ Homo sapiens $\mathrm{GN}=\mathrm{PTGIS} \mathrm{PE}=1 \mathrm{SV}=1$ \\
\hline P63267 & ACTH_HUMAN & Actin gamma-enteric smooth muscle $\mathrm{OS}=$ Homo sapiens $\mathrm{GN}=\mathrm{ACTG} 2 \mathrm{PE}=1 \mathrm{SV}=1$ \\
\hline Q15326 & ZMY11_HUMAN & Zinc finger MYND domain-containing protein $11 \mathrm{OS}=$ Homo sapiens $\mathrm{GN}=\mathrm{ZMYND} 11 \mathrm{PE}=1 \mathrm{SV}=2$ \\
\hline P55058 & PLTP_HUMAN & Phospholipid transfer protein OS $=$ Homo sapiens $\mathrm{GN}=\mathrm{PLTP} \mathrm{PE}=1 \mathrm{SV}=1$ \\
\hline P50440 & GATM_HUMAN & Glycine amidinotransferase mitochondrial $\mathrm{OS}=$ Homo sapiens $\mathrm{GN}=\mathrm{GATM} P \mathrm{PE}=1 \mathrm{SV}=1$ \\
\hline Q8IWL1 & SFPA2_HUMAN & Pulmonary surfactant-associated protein $\mathrm{A} 2 \mathrm{OS}=$ Homo sapiens $\mathrm{GN}=\mathrm{SFTPA} 2 \mathrm{PE}=1 \mathrm{SV}=1$ \\
\hline 000422 & SAP18_HUMAN & Histone deacetylase complex subunit SAP18 OS = Homo sapiens GN = SAP18 PE $=1 \mathrm{SV}=1$ \\
\hline Q86XX4 & FRAS1_HUMAN & Extracellular matrix protein $\mathrm{FRAS1} \mathrm{OS}=$ Homo sapiens $\mathrm{GN}=\mathrm{FRAS1} \mathrm{PE}=1 \mathrm{SV}=2$ \\
\hline P30566 & PUR8_HUMAN & Adenylosuccinate lyase $\mathrm{OS}=$ Homo sapiens $\mathrm{GN}=\mathrm{ADSL} P E=1 \mathrm{SV}=2$ \\
\hline P17661 & DESM_HUMAN & Desmin OS $=$ Homo sapiens $\mathrm{GN}=\mathrm{DES} P \mathrm{PE}=1 \mathrm{SV}=3$ \\
\hline Q06828 & FMOD_HUMAN & Fibromodulin $\mathrm{OS}=$ Homo sapiens $\mathrm{GN}=\mathrm{FMOD} \mathrm{PE}=1 \mathrm{SV}=2$ \\
\hline
\end{tabular}

Table 3 Enlists the proteins identified only in LN metastatic patient sample through mass spectrometric studies.

Protein list-metastasis only

\begin{tabular}{|c|c|c|}
\hline Accession & Entry & Description \\
\hline Q04446 & GLGB_HUMAN & 14-alpha-glucan-branching enzyme OS $=$ Homo sapiens $\mathrm{GN}=\mathrm{GBE} 1 \mathrm{PE}=1 \mathrm{SV}=3$ \\
\hline Q96C23 & GALM_HUMAN & Aldose 1-epimerase $\mathrm{OS}=$ Homo sapiens $\mathrm{GN}=\mathrm{GALM} P E=1 \mathrm{SV}=1$ \\
\hline Q96CN7 & ISOC1_HUMAN & Isochorismatase domain-containing protein $1 \mathrm{OS}=$ Homo sapiens $\mathrm{GN}=\mathrm{ISOC} 1 \mathrm{PE}=1 \mathrm{SV}=3$ \\
\hline Q06124 & PTN11_HUMAN & Tyrosine-protein phosphatase non-receptor type $11 \mathrm{OS}=$ Homo sapiens $\mathrm{GN}=\mathrm{PTPN} 11 \mathrm{PE}=1 \mathrm{SV}=2$ \\
\hline P32456 & GBP2_HUMAN & Guanylate-binding protein $2 \mathrm{OS}=$ Homo sapiens $\mathrm{GN}=\mathrm{GBP} 2 \mathrm{PE}=1 \mathrm{SV}=3$ \\
\hline Q9NVE7 & PANK4_HUMAN & Pantothenate kinase $4 \mathrm{OS}=$ Homo sapiens $\mathrm{GN}=\mathrm{PANK} 4 \mathrm{PE}=1 \mathrm{SV}=1$ \\
\hline Q53RD9 & FBLN7_HUMAN & Fibulin-7 OS $=$ Homo sapiens GN $=\mathrm{FBLN7} P E=2 \mathrm{SV}=1$ \\
\hline Q9UDY2 & ZO2_HUMAN & Tight junction protein $\mathrm{ZO}-2 \mathrm{OS}=$ Homo sapiens $\mathrm{GN}=\mathrm{TJP} 2 \mathrm{PE}=1 \mathrm{SV}=2$ \\
\hline P35237 & SPB6_HUMAN & Serpin B6 OS $=$ Homo sapiens GN $=$ SERPINB6 PE $=1 \mathrm{SV}=3$ \\
\hline P07358 & CO8B_HUMAN & Complement component $\mathrm{C} 8$ beta chain $\mathrm{OS}=$ Homo sapiens $\mathrm{GN}=\mathrm{C} 8 \mathrm{~B} \mathrm{PE}=1 \mathrm{SV}=3$ \\
\hline Q6RW13 & ATRAP_HUMAN & Type- 1 angiotensin II receptor-associated protein $\mathrm{OS}=$ Homo sapiens $\mathrm{GN}=\mathrm{AGTRAP} P \mathrm{PE}=1 \mathrm{SV}=1$ \\
\hline Q8WXX5 & DNJC9_HUMAN & DnaJ homolog subfamily $\mathrm{C}$ member $9 \mathrm{OS}=$ Homo sapiens $\mathrm{GN}=$ DNAJC9 PE $=1 \mathrm{SV}=1$ \\
\hline Q16625 & OCLN_HUMAN & Occludin OS $=$ Homo sapiens $\mathrm{GN}=\mathrm{OCLN} P E=1 \mathrm{SV}=1$ \\
\hline Q6P2P2 & ANM9_HUMAN & Putative protein arginine $\mathrm{N}$-methyltransferase $9 \mathrm{OS}=$ Homo sapiens $\mathrm{GN}=\mathrm{PRMT9} P \mathrm{PE}=2 \mathrm{SV}=1$ \\
\hline P27361 & MK03_HUMAN & Mitogen-activated protein kinase $3 \mathrm{OS}=$ Homo sapiens $\mathrm{GN}=\mathrm{MAPK} 3 \mathrm{PE}=1 \mathrm{SV}=4$ \\
\hline Q9UKG1 & DP13A_HUMAN & DCC-interacting protein 13 -alpha OS $=$ Homo sapiens $\mathrm{GN}=\mathrm{APPL} 1 \mathrm{PE}=1 \mathrm{SV}=1$ \\
\hline P17301 & ITA2_HUMAN & Integrin alpha-2 OS $=$ Homo sapiens $\mathrm{GN}=\mathrm{ITGA} 2 \mathrm{PE}=1 \mathrm{SV}=1$ \\
\hline Q49A26 & GLYR1_HUMAN & Putative oxidoreductase GLYR1 OS $=$ Homo sapiens $\mathrm{GN}=\mathrm{GLYR} 1 \mathrm{PE}=1 \mathrm{SV}=3$ \\
\hline Q9BXR6 & FHR5_HUMAN & Complement factor $\mathrm{H}$-related protein $5 \mathrm{OS}=$ Homo sapiens $\mathrm{GN}=\mathrm{CFHR5} \mathrm{PE}=1 \mathrm{SV}=1$ \\
\hline
\end{tabular}

(4499S, CST), anti-actin (ab49900, CST), anti-Na-K ATPase (MA3-928, Thermo Fisher Scientific), anti-HUWE1 (A300-486A, Bethyl), anti-USP7 (A300-033A, Bethyl), anti-USP9X (A301-351A, Bethyl) and anti-KRAS (c-166691, Santa Cruz).

In vitro kinase assay. $2 \times 10^{6} \mathrm{HeLa}$ cells were seeded in $100 \mathrm{~mm}$-cell culture plates and V5-tagged plasmids were transfected using PEI (as described in Cell culture and transient transfection) on the following day. $48 \mathrm{~h}$ post transfection, cells were lysed in lysis buffer $(250 \mathrm{mM} \mathrm{NaCl}, 50 \mathrm{mM}$ Tris-HCl pH 7.5, $10 \%$ glycerol, $1 \%$ Triton X-100 with protease inhibitor cocktail) and V5-tagged proteins were immunoprecipitated using V5 antibody and immobilized to agarose-coupled protein A/G beads (Roche, cat. nos. 11-134-515-001 and 11-243-233-001) overnight. Protein bound beads were washed with the lysis buffer and used for in vitro kinase assay. Kinase assay was performed using dephosphorylated myelin basic protein (MBP) (13-110, Merck) as a substrate in $25 \mathrm{mM}$ Tris (pH 7.5), $5 \mathrm{mM} \beta$ glycerolphosphate, $2 \mathrm{mM}$ DTT, $0.1 \mathrm{mM} \mathrm{Na} \mathrm{VO}_{4}, 10 \mathrm{mM} \mathrm{MgCl}$ and $5 \mathrm{mM}$ ATP (Enzo, BML-EW9805-0100) in a final volume of $40 \mu \mathrm{L}$. The kinase assay mixture was incubated with the immunoprecipitated V5-tagged proteins for $30 \mathrm{~min}$ at $30^{\circ}$ C. The kinase reaction was terminated by adding $20 \mu \mathrm{L}$ SDS-sample buffer $(0.125$ M Tris-HCl, pH 6.8, 4\% SDS, 10\% glycerol, $10 \mathrm{mM}$ DTT and bromophenol blue) followed by boiling at $95^{\circ} \mathrm{C}$ for $10 \mathrm{~min}$. The samples were loaded onto $12 \%$ SDS-PAGE gels and subjected to immunoblotting analysis.
Cell viability assay (MTT assay). Cell viability assay was performed by employing the Cell Proliferation Kit I (11465007001, Roche). Nthy-ori 3-1 cells stably expressing pPHAGE C-TAP empty vector and pPHAGE C-TAP TFG-RET were seeded in 96-well cell culture plates in $100 \mu \mathrm{L}$ of complete growth medium $(10,000$ cells per well, triplicates per condition). Fold change was calculated as: Fold change $=($ O.D. at $48 \mathrm{~h}$ or $72 \mathrm{~h}$-background $) /($ O.D. at $2 \mathrm{~h}$-background $)$. In case of siRNA-transfected cells, cells were harvested from 6-well cell culture plates $24 \mathrm{~h}$ post transfection, counted and then seeded in 96-well cell culture plates. Cellular viability was assessed after 2, 48 and $72 \mathrm{~h}$ by adding $10 \mu \mathrm{L}$ of MTT solution for 2-4 h. After incubation, $100 \mu \mathrm{L}$ of solubilization buffer was added to each well and incubated overnight in $\mathrm{CO}_{2}$ incubator. Absorbance of the solubilized MTT was measured by absorbance plate reader (O.D. at $570 \mathrm{~nm}$ ). For each condition, the average of three replicates was calculated. Then, the percentage of viable cells was determined as: Percentage of viable cells $=100^{*}(\mathrm{OD}$ of siRNA - OD of the background $) /(\mathrm{OD}$ of sicontrol - OD of the background).

Soft agar colony formation assay. $1.5 \%$ agarose solution was mixed with $2 \times$ growth medium (with $20 \%$ FCS, $2 \times$ inhibitor) to get a final mixture with $0.75 \%$ agarose in $1 \times$ growth medium (bottom agar medium). $1.5 \mathrm{~mL}$ of this bottom agar medium was added per well in a 6-well plate and incubated at room temperature for at least $10 \mathrm{~min}$ to solidify agarose. Nthy-ori 3-1 cells stably expressing pPHAGE C-TAP empty vector and pPHAGE C-TAP TFG-RET were diluted in $2 \times$ growth 
a
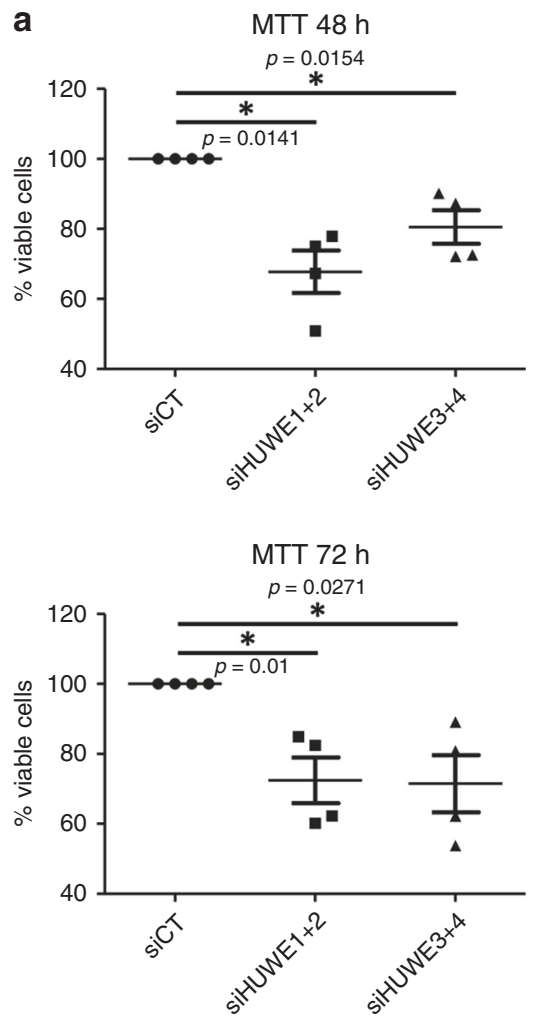

b

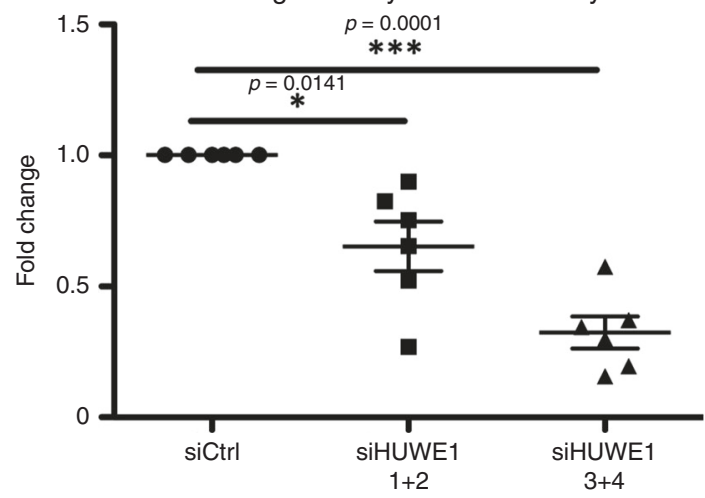

EdU $48 \mathrm{~h}$
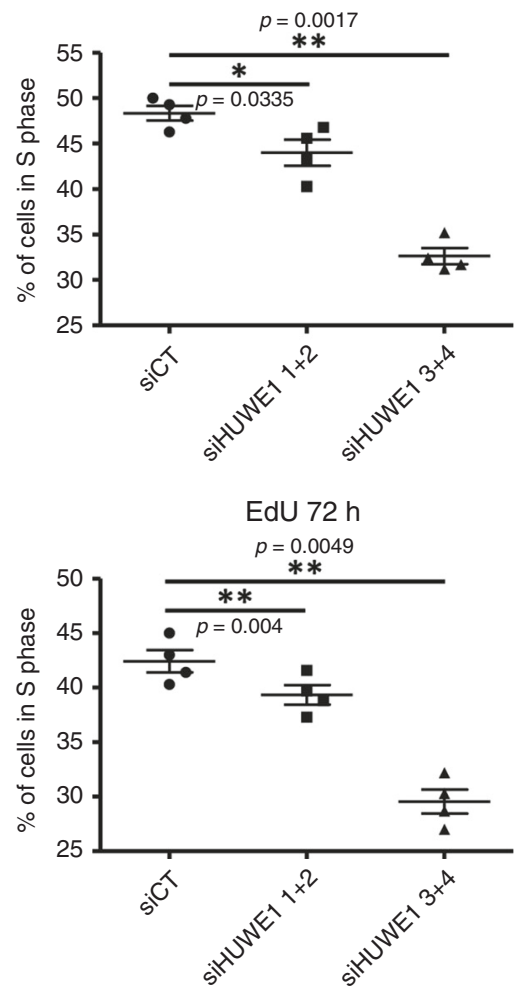

Cell number $48 \mathrm{~h}$

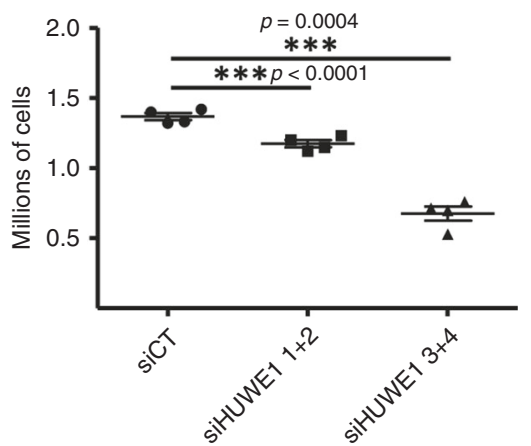

Cell number $72 \mathrm{~h}$

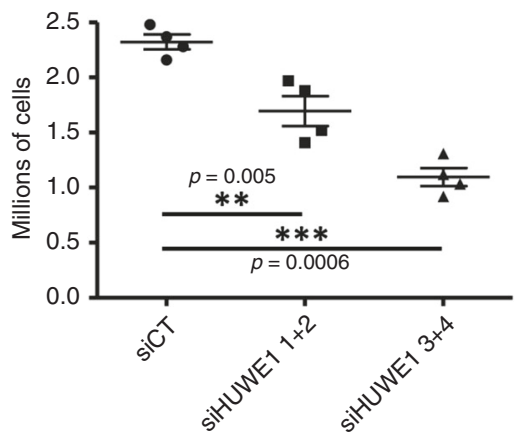

C

Soft agar colony formation assay

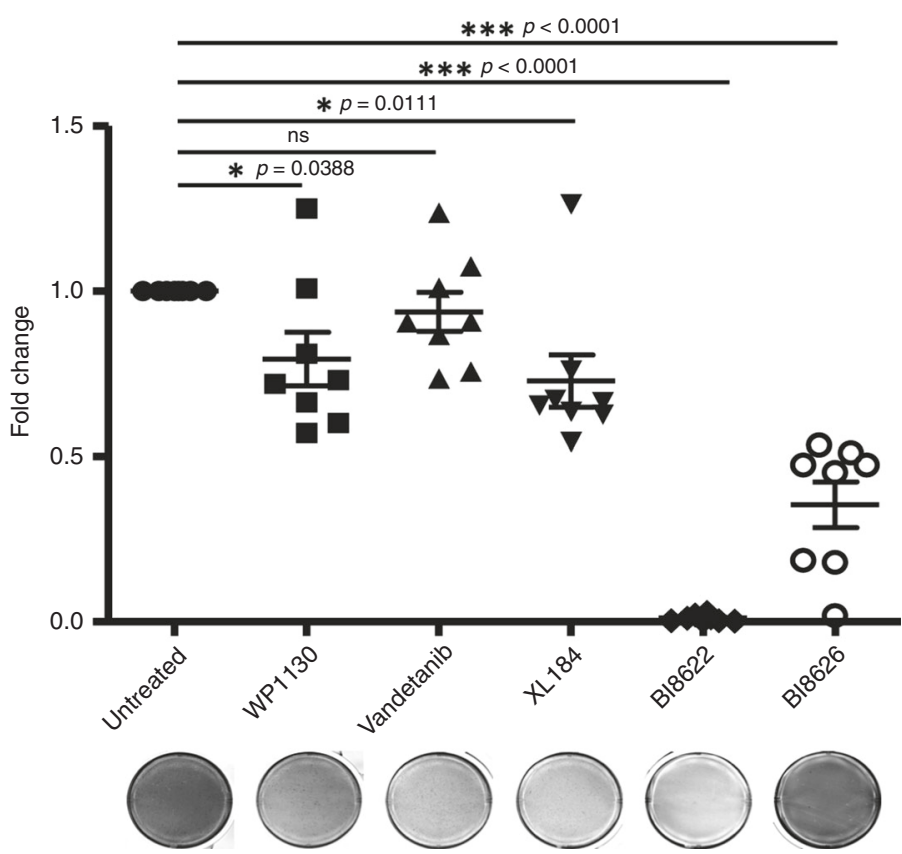

medium and mixed with $0.9 \%$ agarose solution to a final concentration of $0.45 \%$ agarose. $1.5 \mathrm{~mL}$ of this cell suspension was added on the bottom agarose layer (5000 cells/condition). In case of siRNA-transfected cells, the cells were harvested $24 \mathrm{~h}$ post siRNA transfection from 6-well plates and then seeded for the soft agar assay. The cells seeded in soft agar were cultured for 2-4 weeks with addition of $100 \mu \mathrm{L}$ of complete medium (with inhibitor) twice weekly. The colonies were stained with $0.02 \%$ crystal violet solution by gentle agitation at room temperature for about 30 min followed by washes with water. The images were taken with a ChemiDoc Touch (Bio-Rad) imaging system and the number of colonies was counted by Image J software. The anchorage independent growth of TFG-RET transformed cells was also tested and quantified in the presence of multiple inhibitors (WP1130 $1 \mu \mathrm{M}$, Vandetanib $1 \mu \mathrm{M}$, XL184 $2.5 \mu \mathrm{M}$, BI8622 $20 \mu \mathrm{M}$, BI8626 $20 \mu \mathrm{M})$.
Dimerization experiments. RET wild type, TFG-RET and TFG-RET mutant constructs in pPHAGE C-TAP (FLAG, HA tagged) and pcDNA3 Dest 40 (V5, Histagged) were co-transfected as indicated in the results in HeLa cells using PEI (see Cell culture and transient transfection). $48 \mathrm{~h}$ post transfection, cells were lysed in lysis buffer ( $250 \mathrm{mM} \mathrm{NaCl}, 50 \mathrm{mM}$ Tris-HCl pH 7.5, $10 \%$ glycerol, $1 \%$ Triton X100 with protease inhibitor cocktail) and FLAG-tagged protein was immunoprecipitated using FLAG beads (Anti-FLAG ${ }^{\circledR}$ M2 Affinity Gel, A2220-5ML, Sigma). The co-precipitation of V5-tagged proteins was tested by immunoblots.

Heteromerization studies. Nthy-ori 3-1 cells stably expressing pPHAGE C-TAP RET wild type and pPHAGE C-TAP TFG-RET were seeded in 6-well cell culture 
Fig. 6 Inhibition of ubiquitination-associated proteins leads to reduced cell viability and transformation of TFG-RET expressing cells. a HUWE1 was transiently knocked down using siRNA in Nthy-TFG-RET cells. The cells were seeded in 6-well and 96-well plates and subjected to EdU, cell counting and MTT assay after 48 and $72 \mathrm{~h}$, respectively. Knockdown of HUWE1 resulted in a significant reduction of cell viability (MTT), proliferation (EdU) and cell number (cell counting). Error bars represent \pm SEM $(n=4)$. Paired $t$-test, two-tailed, $p$-values: ${ }^{*}<0.05,{ }^{* *}<0.01$ and ${ }^{* \star *}<0.0001$. For FACS analysis, the gating strategy is depicted in Supplementary Fig. 3A. For MTT assays, the percentages of viable cells are shown. For EdU assays, the percentages of cells in $S$ phase are depicted. In the cell-counting experiments, the absolute viable cell numbers are shown. $\mathbf{b}$ HUWE1 was transiently knocked down using siRNA in Nthy-TFG-RET cells and cultured in soft agar for 2 weeks followed by staining with crystal violet. HUWE1 knockdown resulted in a significant reduction in the number of colonies. Error bars represent \pm SEM. Three independent experiments with technical duplicates were performed $(n=3)$. Paired $t$-test, twotailed, $p$-values: ${ }^{\star}<0.05,{ }^{\star * *}<0.0001$. c Tyrosine kinase, DUB and HUWE1 inhibitors reduce oncogenic growth in TFG-RET expressing cells-soft agar colony formation assay. Nthy-ori 3-1 cells stably expressing TFG-RET were treated with inhibitors with indicated concentrations. Error bars represent \pm SEM $(n=3)$. Paired $t$-test, two-tailed, $p$-value: ${ }^{*}<0.05,{ }^{* *}<0.01,{ }^{* \star *}<0.0001$, ns not significant. In $\mathbf{b}$ and $\mathbf{c}$ the fold changes in the number of viable colonies from the analyzed samples are presented.

Table 4 Summarizes the $I C_{50}$ values and effects/uses of the inhibitors used in the experiments.

\section{List of inhibitors and targets/functions}

\begin{tabular}{lll}
\hline Compound & IC $_{\mathbf{5 0}}$ & Description \\
\hline WP1130 & $1.25-5 \mu \mathrm{M}$ (Usp9x) & Deubiquitinase inhibitor \\
XL184 (Cabozantinib) & $4 \mathrm{nM}$ (RET) & RTK inhibitor, used in the treatment of MTC, RCC \\
Vandatenib & $40 \mathrm{nM}$ (VEGFR2) 110 nM (VEGFR3) 500 nM (EGFR) & TK inhibitor, used in the treatment of MTC \\
BI8622 & $3.1 \mu \mathrm{M}$ (HUWE1) & HUWE1 inhibitor \\
Bl8626 $2.5 \mu \mathrm{M}$ & $2.5 \mu \mathrm{M}$ (HUWE1) & HUWE1 inhibitor \\
\hline
\end{tabular}

RTK receptor tyrosine kinase, TK tyrosine kinase, MTC medullary thyroid cancer, $R C C$ renal cell carcinoma.

plates. Upon becoming $70 \%$ confluent, cells were treated either with DMSO (A3672.0250 Applichem), Dithio-bismaleimidoethane (DTME) alone (0.2 mM, $1 \mathrm{~h}$; 22335, Thermo Fisher Scientific) or DTME followed by Dithiothreitol (DTT) (100 $\mathrm{mM}, 15 \mathrm{~min}$ ). After thorough washes with PBS, cells were lysed in sample buffer (cells treated with only DTME were lysed in non-DTT containing sample buffer) and subjected to immunoblot analysis.

Imaging studies. Nthy-ori 3-1 cells stably expressing FLAG-tagged pPHAGE CTAP TFG-RET or pPHAGE C-TAP RET were seeded on glass coverslips. The cells were transiently transfected with EGFP-C1 Lck-GFP (61099, Addgene) using PEI (as described in Cell culture and transient transfection). $48 \mathrm{~h}$ later, the cells were fixed using $4 \%$ formaldehyde for $10 \mathrm{~min}$ after media removal and two PBS washes. The cells were permeabilized using $0.1 \%$ Triton X-100 (3 min, room temperature). After two subsequent washes with PBS, the cells were blocked with $1 \%$ BSA for 30 min at room temperature. The cells were then stained for TFG-RET/RET using anti-FLAG $^{\circledast}$ M2-Peroxidase (A8592, Sigma, 1:500 dilution in 1\% BSA) for $1 \mathrm{~h}$ at room temperature. The cells were then washed with PBS and stained with antimouse Cy3 antibody (1:100 dilution in 1\% BSA) along with Hoechst $(2.5 \mu \mathrm{g} / \mathrm{mL}$ in $1 \% \mathrm{BSA}$ ) for $30 \mathrm{~min}$ in the dark at room temperature. The cells were washed with PBS and mounted on glass slides with Mowiol (+DABCO). Cells were imaged using a Leica SP8 confocal microscope $(\times 63$, oil immersion objective, Cy3 excitation at $552 \mathrm{~nm}$, GFP excitation at $488 \mathrm{~nm})$.

Mice. Female NOD.CB17-Prkscid mice were obtained from Janvier. All animals were housed at the animal facility of Johannes Gutenberg University using institutionally approved protocols (Landesuntersuchungsamt Koblenz). Animal procedures were performed under the supervision of the authorized investigators in accordance with the European Union normative for care and use of experimental animals.

Subcutaneous tumor model. Nthy-ori-3 cells or Nthy-TFG-RET $\left(4 \times 10^{6}\right.$ cells in $200 \mu \mathrm{L}$ PBS) were injected subcutaneously into the flank of 8- to 10-week-old female NOD.CB17-Prkscid mice. Tumor growth was observed over 115 days. Tumor sizes were determined by caliper measurements every other day. Tumor volume was calculated using the following formula: Volume $=($ width $\times$ length $) / 2$. Exclusion criteria were tumor volumes exceeding $1 \mathrm{~cm}^{3}$, tumor sizes more than 1.5 $\mathrm{cm}$ in any direction and necrosis.

Gel filtration. HeLa cells were transiently transfected with TFG-RET, TFG-RET K14ER22ER23E and TFG-RET $\triangle 97-124$ in pcDNA3 Dest 40 (V5-, His-tagged). Approximately $1.5 \times 10^{7}$ cells were lysed $48 \mathrm{~h}$ post transfection in $500 \mu \mathrm{L}$ lysis buffer ( $50 \mathrm{mM}$ Tris pH 7.5, $150 \mathrm{mM} \mathrm{NaCl}, 10 \%$ glycerol, protease inhibitor cocktail (539131, Calbiochem)) by sonication ( $-50 \%$ amplitude, $5 \mathrm{~s}$, four cycles). Cell lysates were subjected to ultracentrifugation $\left(30,000 \times g\right.$ for $1 \mathrm{~h}$ at $\left.4{ }^{\circ} \mathrm{C}\right)$ and the supernatant (cytosolic fraction) was separated by size exclusion chromatography using an ÄKTA-Pure25 system equipped with two Superose-6 HR-10/30 columns (GE Healthcare, Freiburg, Germany) connected in series. The columns were preequilibrated with running buffer $(50 \mathrm{mM}$ Tris $\mathrm{pH} 7.5,150 \mathrm{mM} \mathrm{NaCl}$ buffer, filtered $0.22 \mu \mathrm{m}) .500 \mu \mathrm{L}$ of cytosolic fraction were injected and separated at a flow rate of $0.5 \mathrm{~mL} / \mathrm{min}$. Fractions of $500 \mu \mathrm{L}$ were collected and subjected to immunoblot analysis. Molecular weight reference proteins (Gel Filtration Marker Kit (mwgf1000, Sigma-Aldrich)) were separated under identical conditions and a calibration curve was obtained by plotting molecular weight (log scale) against elution volume.

In-solution digestion and filter-aided sample preparation (FASP). Samples for initial proteomic experiments were processed by in-solution digestion: Four volumes of ice cold acetone were added to the protein lysates (prepared as mentioned in the previous section (isolation of protein, DNA and RNA from patient tissue)), vortexed and precipitated at $-20^{\circ} \mathrm{C}$ overnight. Samples were centrifuged at $16,000 \times g$ for $20 \mathrm{~min}$ at $4^{\circ} \mathrm{C}$ and the supernatant was discarded. Proteins were re-dissolved in $50 \mu \mathrm{L} 6 \mathrm{M}$ urea and $100 \mathrm{mM}$ ammonium bicarbonate, $\mathrm{pH}$ 7.8. For reduction and alkylation of cysteines, $2.5 \mu \mathrm{L}$ of $200 \mathrm{mM}$ DTT in $100 \mathrm{mM}$ Tris- $\mathrm{HCl}$, $\mathrm{pH} 8$ was added and the samples were incubated at $37^{\circ} \mathrm{C}$ for $1 \mathrm{~h}$ followed by addition of $7.5 \mu \mathrm{L} 200 \mathrm{mM}$ iodoacetamide for $1 \mathrm{~h}$ at room temperature in the dark. The alkylation reaction was quenched by adding $10 \mu \mathrm{L} 200 \mathrm{mM}$ DTT at $37^{\circ} \mathrm{C}$ for 1 h. Subsequently, the proteins were digested with $10 \mu \mathrm{g}$ trypsin GOLD (Promega) for $16 \mathrm{~h}$ at $37^{\circ} \mathrm{C}$. The digestion was stopped by adding $5 \mu \mathrm{L} 50 \%$ formic acid and the generated peptides were purified using OMIX C18, $10 \mu \mathrm{L}$ (Agilent, Santa Clara), and dried using a Speed Vac concentrator (Concentrator Plus, Eppendorf).

The second proteomic dataset was processed using filter-aided sample preparation (FASP) as detailed before ${ }^{42,43}$. In brief, cells were dissolved in a buffer containing $7 \mathrm{M}$ urea, $2 \mathrm{M}$ thiourea, $5 \mathrm{mM}$ DTT, 2\% (w/v) CHAPS and lysed by sonication at $4{ }^{\circ} \mathrm{C}$ for $15 \mathrm{~min}$ using a Bioruptor (Diagenode, Liège, Belgium). The protein concentration was determined using the Pierce $660 \mathrm{~nm}$ protein assay (Thermo Fisher Scientific) according to the manufacturer's protocol. $20 \mu \mathrm{g}$ of total protein were used for FASP. Proteins were transferred onto spin filter columns (Nanosep centrifugal devices with Omega membrane, $30 \mathrm{kDa}$ MWCO; Pall, Port Washington, NY) and detergents were removed washing the samples three times with a buffer containing $8 \mathrm{M}$ urea. After reduction and alkylation by DTT and iodoacetamide (IAA), excess IAA was quenched with DTT and the membrane was washed three times with $50 \mathrm{mM} \mathrm{NH}_{4} \mathrm{HCO}_{3}$. Afterwards, proteins were digested overnight at $37^{\circ} \mathrm{C}$ with trypsin (Trypsin Gold, Promega, Madison, WI) using an enzyme-to-protein ratio of 1:50 (w/w). After digestion, peptides were recovered by centrifugation and two additional washes with $50 \mathrm{mM} \mathrm{NH}_{4} \mathrm{HCO}_{3}$. Combined flowthroughs were acidified with trifluoroacetic acid (TFA) to a final concentration of 
$1 \%(\mathrm{v} / \mathrm{v})$ TFA and lyophilized. Purified peptides were reconstituted in $0.1 \%(\mathrm{v} / \mathrm{v})$ formic acid (FA) for LC-MS analysis.

Liquid chromatography-mass spectrometry (LC-MS). The tryptic peptides were dissolved in $10 \mu \mathrm{L} 0.1 \%$ formic acid $/ 2 \%$ acetonitrile and $5 \mu \mathrm{L}$ were analyzed using an Ultimate 3000 RSLCnano-UHPLC system connected to a Q Exactive mass spectrometer (Thermo Fisher Scientific) equipped with a nano-electrospray ion source. For liquid chromatography separation, an Acclaim PepMap 100 column (C18, $2 \mu \mathrm{m}$ beads, $100 \AA$, $75 \mu \mathrm{m}$ inner diameter, $50 \mathrm{~cm}$ length) (Dionex, Sunnyvale CA, USA) was used. A flow rate of $300 \mathrm{~nL} / \mathrm{min}$ was employed with a solvent $\mathrm{B}$ gradient of $4-35 \%$ in $180 \mathrm{~min}$. Solvent A was $0.1 \%$ formic acid and solvent B was $0.1 \%$ formic acid $/ 90 \%$ acetonitrile. The mass spectrometer was operated in the data-dependent mode to automatically switch between MS and MS/MS acquisition. Survey full scan MS spectra (from $\mathrm{m} / z 400$ to 2000) were acquired with the resolution $R=70,000$ at $\mathrm{m} / z 200$, after accumulation to a target of 1e6. The maximum allowed ion accumulation times were $60 \mathrm{~ms}$. The method used allowed sequential isolation of up to the ten most intense ions, depending on signal intensity (intensity threshold 1.7e4), for fragmentation using higher-energy collisional induced dissociation (HCD) at a target value of 1e5 charges, NCE 28, and a resolution $R=17,500$. Target ions already selected for MS/MS were dynamically excluded for $30 \mathrm{~s}$. The isolation window was $m / z=2$ without offset. For accurate mass measurements, the lock mass option was enabled in MS mode.

For label-free quantification analysis, raw data were imported into PEAKS v8.5 (Bioinformatics Solutions Inc, Toronto, CA). Processed raw data were searched in PEAKS against the UniProt SwissProt database (Human, 20,279 proteins) assuming the digestion enzyme trypsin, at maximum two missed cleavage sites, parent ion tolerance of $10 \mathrm{ppm}$, fragment ion mass tolerance of $0.02 \mathrm{Da}$, carbamidomethylation of cysteines as fixed modification, and oxidation of methionines, deamidation of asparagine and glutamine residues as variable modifications. Label-free quantification was performed in the PEAKS software using a maximum mass difference of $15 \mathrm{ppm}$ and a maximum retention time difference of $1.5 \mathrm{~min}$ for clustering and a $0.1 \%$ FDR threshold for peak annotation. For relative quantification, the data were normalized based on total ion current (TIC) in the PEAKS software to correct for unequal sample loading.

Proteomic samples (Patients \#2, \#3, \#4, \#5) prepared by FASP were analyzed by LC-MS on a Synapt G2-S HDMS mass spectrometer (Waters Corporation) coupled to a nanoAcquity UPLC system (Waters Corporation). Water containing $0.1 \%(\mathrm{v} / \mathrm{v}) \mathrm{FA}, 3 \%(\mathrm{v} / \mathrm{v})$ dimethyl sulfoxide (DMSO) served as mobile phase A and acetonitrile (ACN) containing 0.1\% FA (v/v), 3\% (v/v) DMSO as mobile phase $\mathrm{B}^{44}$. Tryptic peptides (corresponding to $200 \mathrm{ng}$ ) were loaded onto an HSS-T3 C18 1.8 $\mu \mathrm{m}, 75 \mu \mathrm{m} \times 250 \mathrm{~mm}$ reverse-phase column from Waters Corporation in direct injection mode. Peptides were separated at a flow rate of $300 \mathrm{~nL} / \mathrm{min}$ applying a gradient from 5 to $40 \%$ (v/v) mobile phase B over $90 \mathrm{~min}$. Afterwards, the column was washed with $90 \%$ mobile phase B and re-equilibrated to initial conditions resulting in a total analysis time of $120 \mathrm{~min}$. The column was heated to $55^{\circ} \mathrm{C}$. Eluting peptides were analyzed in positive mode ESI-MS by ion-mobility separation (IMS) enhanced data-independent acquisition (DIA) UDMS ${ }^{\mathrm{E}}$ mode as described before ${ }^{43,45}$. Acquired MS data were post-acquisition lock mass corrected using [Glu1]-Fibrinopeptide B, which was sampled every $30 \mathrm{~s}$ into the mass spectrometer via the reference sprayer of the NanoLockSpray source at a concentration of $250 \mathrm{fmol} / \mu \mathrm{L}$.

LC-MS DIA raw data were processed and searched with ProteinLynx Global SERVER (PLGS) (version 3.02 build 5, Waters Corporation) against a custom compiled database containing UniProtKB/SwissProt entries of the human reference proteomes (entries: 20,394) as well as common contaminants. Following search criteria were applied: (i) Trypsin as digestion enzyme allowing up to two missed cleavages, (ii) carbamidomethyl cysteine was defined as fixed and (iii) methionine oxidation as variable modification. The false discovery rate (FDR) for peptide and protein identification was assessed searching a reversed database and set to a $1 \%$ threshold for database search in PLGS. Label-free quantification analysis was performed using ISOQuant as described before ${ }^{45}$. For each protein, absolute insample amounts were estimated using TOP 3 quantification ${ }^{46}$. The mass spectrometry proteomics data have been deposited to the ProteomeXchange consortium PRIDE under two data set identifiers (a) PXD016828 (patient \#1) and (b) PXD016739 (patients \#2-\#5).

Endogenous ubiquitination experiments. Nthy-ori 3-1 cells stably expressing pPHAGE C-TAP TFG-RET (in $100 \mathrm{~mm}$-cell culture dishes, about 70\% confluent) were treated either with DMSO, $20 \mu \mathrm{M}$ BI8622 or BI8626 (synthesized by Syngene International limited, India) inhibitors for $2 \mathrm{~h}$ followed by treatment with $10 \mu \mathrm{M}$ MG132 for $5 \mathrm{~h}$ at $37^{\circ} \mathrm{C}$. Following treatment, cells were lysed in lysis buffer and $250 \mu \mathrm{g}$ protein was used to isolate ubiquitinated protein using UBIQAPTURE-Q ${ }^{\circledR}$ kit (BML-UW8995-0001, Enzo) according to the manufacturer's protocol. Ubiquitination of proteins was determined by immunoblot analysis.

Cellular fractionation assay. Nthy-ori 3-1 cells stably expressing pPHAGE CTAP RET wild type and pPHAGE C-TAP TFG-RET were cultured in $100 \mathrm{~mm}$ dishes. $48 \mathrm{~h}$ post seeding, the growth media were removed from the culture dish and the cells were washed with cold PBS. Cells were subsequently lysed using buffers from ProteoExtract ${ }^{\circledR}$ Subcellular Proteome Extraction Kit (539790, Merck) according to the manufacturer's protocol. Lysates collected were subjected to immunoblot analysis.

Cycloheximide-chase assay. Nthy-ori 3-1 cells were transiently transfected using PEI (as described earlier) with different plasmids in $100 \mathrm{~mm}$ dishes. $24 \mathrm{~h}$ post transfection, cells were re-seeded in 12-well plates. After $24 \mathrm{~h}$, cycloheximide (100 $\mu \mathrm{g} / \mathrm{mL}$ ) was added to the cells and samples were collected in SDS-sample buffer at indicated time points and boiled at $95^{\circ} \mathrm{C}$ for $10 \mathrm{~min}$. Cell lysates were subjected to immunoblot analysis.

EdU DNA synthesis assay. Nthy-ori 3-1 cells stably expressing pPHAGE C-TAP empty vector, $p$ PHAGE C-TAP TFG-RET or pPHAGE C-TAP RET were seeded in $60 \mathrm{~mm}$-cell culture plates $\left(0.4 \times 10^{6}\right.$ cells per plate). EdU DNA synthesis assay was performed using Click-iT ${ }^{\circledR}$ Plus EdU Pacific Blue ${ }^{\text {tut }}$ Flow Cytometry Assay Kit (Thermo Fisher Scientific, C10636) according to the manufacturer's protocol. Briefly, $72 \mathrm{~h}$ post seeding, the cells were incubated for $2 \mathrm{~h}$ in $10 \mu \mathrm{M}$ EdU (5ethynyl-2'-deoxyuridine). The cells were then harvested, fixed, permeabilized and stained with pacific blue ligand (for incorporated EdU) and propidium iodide. Subsequently, pacific blue-positive cells were measured using a flow cytometer (405 nm laser). For EdU assays in cells after transient siRNA knock down, cells were seeded in $60 \mathrm{~mm}$-cell culture plates and transfected with siRNA on the next day (as described in siRNA transfection). Total cell number was determined using an automated cell counter (Bio-Rad TC20 ${ }^{\mathrm{TM}}$ Automated Cell Counter, 1450102) to normalize the starting cell numbers. EdU DNA synthesis assay was performed $48 \mathrm{~h}$ and $72 \mathrm{~h}$ post transfection.

siRNA transfection. ON-TARGET plus Human siRNAs (set of 4 per target) were purchased from Dharmacon (details below). Two sets of siRNAs (each set with 2 different siRNAs) were used for each protein target. Cells were transiently transfected with siRNA using SAINT-sRNA transfection reagent (SR-2003-01, Synvolux Products) according to the manufacturer's protocol. Briefly, cells were seeded at a density of $0.2 \times 10^{6}$ cells per well of a 6 -well plate. On the next day, $200 \mu \mathrm{L}$ PBS, $1.25 \mu \mathrm{L}$ of each of the two siRNAs $(20 \mu \mathrm{M})$ and $20 \mu \mathrm{L}$ of SAINT-sRNA transfection reagent were mixed and incubated in room temperature for $15 \mathrm{~min}$ to form complexes. After changing the medium to serum-free RPMI-1640 medium, the siRNA-SAINT-sRNA complexes were added dropwise to the cells. The medium was replaced with fresh RPMI-1640 containing 10\% FBS. The samples were collected for Western blot analysis $48 \mathrm{~h}$ post transfection.

ON-TARGETplus non-targeting siRNA

siRNA D-001810-01-05 UGGUUUACAUGUCGACUAA

siRNA D-001810-01-06 UGGUUUACAUGUUGUGUGUGA

ON-TARGETplus Human HUWE1 siRNA LQ-007185-00-0005

J-007185-07, HUWE1 GCUUUGGGCUGGCCUAAUA

J-007185-08, HUWE1 GCAGUUGGCGGCUUUCUUA

J-007185-09, HUWE1 GAGCCCAGAUGACUAAGUA

J-007185-10, HUWE1 UAACAUCAAUUGUCCACUU

ON-TARGETplus Human USP7 siRNA LQ-006097-00-0005

J-006097-05, USP7 AAGCGUCCCUUUAGCAUUA

J-006097-06, USP7 GCAUAGUGAUAAACCUGUA

J-006097-07, USP7 UAAGGACCCUGCAAAUUAU

J-006097-08, USP7 GUAAAGAAGUAGACUAUCG

ON-TARGETplus Human USP9X siRNA LQ-006099-00-0005

J-006099-06, USP9X AGAAAUCGCUGGUAUAAAU

J-006099-07, USP9X ACACGAUGCUUUAGAAUUU

J-006099-08, USP9X GUACGACGAUGUAUUCUCA

J-006099-09, USP9X GAAAUAACUUCCUACCGA

Statistical analysis. Data were statistically analyzed by using GraphPad Prism software (Prism 5 for Mac OSX, version 5.0a). Tests employed are mentioned in the figure legends.

Reporting summary. Further information on research design is available in the Nature Research Reporting Summary linked to this article.

\section{Data availability}

The data are available from authors upon request. The proteomics data are available through PRIDE (under accession numbers PXD016739 and PXD016828). Sequencing data were submitted to the EBI ENA database under accession id PRJEB37220 (ERP120526)

Received: 14 December 2018; Accepted: 2 April 2020; Published online: 28 April 2020 


\section{References}

1. National Cancer Institute. SEER Cancer Stat Facts: Thyroid Cancer https:// seer.cancer.gov/statfacts/html/thyro.html (National Cancer Institute, Bethesda, MD).

2. National Cancer Institute. Cancer Stat Facts: Thyroid cancer. https://seer. cancer.gov/statfacts/html/thyro.html (National Cancer Institute, Bethesda, MD).

3. Lim, H. et al. Trends in thyroid cancer incidence and mortality in the United States, 1974-2013. JAMA 317, 1338-1348 (2017).

4. Kilfoy, B. A. et al. Gender is an age-specific effect modifier for papillary cancers of the thyroid gland. Cancer Epidemiol. Biomark. Prev. 18, 1092-1100 (2009).

5. Gilliland, F. D. et al. Prognostic factors for thyroid carcinoma. A populationbased study of 15,698 cases from the Surveillance, Epidemiology and End Results (SEER) program 1973-1991. Cancer 79, 564-573 (1997).

6. Cardis, E. et al. Cancer consequences of the Chernobyl accident: 20 years on. $J$. Radio. Prot. 26, 127-140 (2006).

7. Xing, M. Molecular pathogenesis and mechanisms of thyroid cancer. Nat. Rev. Cancer 13, 184-199 (2013).

8. Luster, M. \& Musholt, T. J. Thyroid gland: thyroid surgery and radioiodine ablation-the surgeon's role. Nat. Rev. Endocrinol. 9, 140-141 (2013).

9. Fedewa, S. A., Jemal, A. \& Chen, A. Y. Trends and predictors of chemotherapy use among thyroid cancer patients in the national cancer database (2004-2013). Eur. Thyroid J. 5, 268-276 (2016).

10. Sun, W. et al. Risk factors for central lymph node metastasis in CN0 papillary thyroid carcinoma: a systematic review and meta-analysis. PLoS ONE 10, e0139021 (2015).

11. Carling, T. \& Udelsman, R. Thyroid cancer. Annu Rev. Med. 65, 125-137 (2014).

12. Williams, D. Cancer after nuclear fallout: lessons from the Chernobyl accident. Nat. Rev. Cancer 2, 543-549 (2002).

13. Nikiforova, M. N. et al. Proximity of chromosomal loci that participate in radiation-induced rearrangements in human cells. Science 290, 138-141 (2000)

14. Santoro, M., Melillo, R. M. \& Fusco, A. RET/PTC activation in papillary thyroid carcinoma. Eur. J. Endocrinol. 155, 645-653 (2006).

15. Cancer Genome Atlas Research Network. Integrated genomic characterization of papillary thyroid carcinoma. Cell 159, 676-690 (2014)

16. Grieco, M. et al. PTC is a novel rearranged form of the ret proto-oncogene and is frequently detected in vivo in human thyroid papillary carcinomas. Cell 60, 557-563 (1990).

17. Moscat, J. et al. Cell signaling and function organized by PB1 domain interactions. Mol. Cell 23, 631-640 (2006).

18. Bienz, M. Signalosome assembly by domains undergoing dynamic head-to-tail polymerization. Trends Biochem Sci. 39, 487-495 (2014).

19. Kawamoto, Y. et al. Identification of RET autophosphorylation sites by mass spectrometry. J. Biol. Chem. 279, 14213-14224 (2004).

20. Myant, K. B. et al. HUWE1 is a critical colonic tumour suppressor gene that prevents MYC signalling, DNA damage accumulation and tumour initiation. EMBO Mol. Med. 9, 181-197 (2017).

21. Peter, S. et al. Tumor cell-specific inhibition of MYC function using small molecule inhibitors of the HUWE1 ubiquitin ligase. EMBO Mol. Med. 6, 1525-1541 (2014).

22. Adhikary, S. et al. The ubiquitin ligase HectH9 regulates transcriptional activation by Myc and is essential for tumor cell proliferation. Cell 123, 409-421 (2005).

23. Zhao, X. et al. The N-Myc-DLL3 cascade is suppressed by the ubiquitin ligase Huwe1 to inhibit proliferation and promote neurogenesis in the developing brain. Dev. Cell 17, 210-221 (2009).

24. Zhong, Q. et al. Mule/ARF-BP1, a BH3-only E3 ubiquitin ligase, catalyzes the polyubiquitination of Mcl-1 and regulates apoptosis. Cell 121, 1085-1095 (2005).

25. Chen, D. et al. ARF-BP1/Mule is a critical mediator of the ARF tumor suppressor. Cell 121, 1071-1083 (2005).

26. Mertens, F. et al. The emerging complexity of gene fusions in cancer. Nat. Rev. Cancer 15, 371-381 (2015).

27. Staubitz, JuliaIsabelle et al. ANKRD26-RET - a novel gene fusion involving RET in papillary thyroid carcinoma. Cancer Genet. 238, 10-17 (2019).

28. Staubitz, J. I. et al. Novel rearrangements involving the RET gene in papillary thyroid carcinoma. Cancer Genet. 230, 13-20 (2019).

29. de Groot, J. W. et al. RET as a diagnostic and therapeutic target in sporadic and hereditary endocrine tumors. Endocr. Rev. 27, 535-560 (2006)

30. Stransky, N. et al. The landscape of kinase fusions in cancer. Nat. Commun. 5, 4846 (2014).

31. Grubbs, E. G. et al. RET fusion as a novel driver of medullary thyroid carcinoma. J. Clin. Endocrinol. Metab. 100, 788-793 (2015).

32. Encinas, M. et al. Tyrosine 981, a novel ret autophosphorylation site, binds c-Src to mediate neuronal survival. J. Biol. Chem. 279, 18262-18269 (2004).
33. Rajalingam, K. \& Dikic, I. SnapShot: expanding the ubiquitin code. Cell 164, 1074-1074 el (2016)

34. Musholt, T. J. et al. Detection of RET rearrangements in papillary thyroid carcinoma using RT-PCR and FISH techniques-a molecular and clinical analysis. Eur. J. Surg. Oncol. 45, 1018-1024 (2018).

35. Li, H. \& Durbin, R. Fast and accurate short read alignment with Burrows Wheeler transform. Bioinformatics 25, 1754-1760 (2009).

36. DePristo, M. A. et al. A framework for variation discovery and genotyping using next-generation DNA sequencing data. Nat. Genet. 43, 491-498 (2011).

37. Saunders, C. T. et al. Strelka: accurate somatic small-variant calling from sequenced tumor-normal sample pairs. Bioinformatics 28, 1811-1817 (2012).

38. Lek, M. et al. Analysis of protein-coding genetic variation in 60,706 humans Nature 536, 285-291 (2016)

39. Wu, T. D. \& Nacu, S. Fast and SNP-tolerant detection of complex variants and splicing in short reads. Bioinformatics 26, 873-881 (2010).

40. Anders, S. \& Huber, W. Differential expression analysis for sequence count data. Genome Biol. 11, R106 (2010).

41. Rudin, C. M. et al. Comprehensive genomic analysis identifies SOX2 as a frequently amplified gene in small-cell lung cancer. Nat. Genet. 44, 1111-1116 (2012).

42. Wisniewski, J. R. et al. Universal sample preparation method for proteome analysis. Nat. Methods 6, 359-362 (2009).

43. Distler, U. et al. Label-free quantification in ion mobility-enhanced dataindependent acquisition proteomics. Nat. Protoc. 11, 795-812 (2016).

44. Hahne, H. et al. DMSO enhances electrospray response, boosting sensitivity of proteomic experiments. Nat. Methods 10, 989-991 (2013).

45. Distler, U. et al. Drift time-specific collision energies enable deep-coverage data-independent acquisition proteomics. Nat. Methods 11, 167-170 (2014).

46. Silva, J. C. et al. Absolute quantification of proteins by LCMSE: a virtue of parallel MS acquisition. Mol. Cell Proteom. 5, 144-156 (2006).

\section{Acknowledgements}

We thank all the patients who have volunteered and donated their biomaterials for the study. We thank Stefanie Wenzel for excellent technical assistance. We thank Dr. Stefanie Zimmer and the biobanking team for their help with the consenting and collection of patient materials. We thank Prof. Dr. Christian Behrends for the pPHAGE vector and Dr. Andreas Ernst for 293T cells. We thank Dr. Christiane Schoenfeld for assisting with the animal experiments and for critical reading of the manuscript. This work is primarily supported from a DKH grant (DKH 70113042) to K.R. and from the CRC 1292 (TP12) and GFK fellowship to K.R. K.R. is supported through a Heisenberg professorship of the DFG (RA1739/4-1). K.R. also acknowledges support from FZI (Forschungszentrum Immuntherapie) of the UMC-Mainz.

\section{Author contributions}

A.K., J.B. and E.R. designed and performed most of the experiments, analyzed/interpreted data and prepared figures. S.S., Z.M., J.W. and E.S. contributed to genomic studies U.D., S.T. and B.T. performed proteomics mass spectrometric analysis. M.F. and M.B. contributed to the PB1 modeling studies, data analysis and manuscript preparation. S.R. performed animal experiments. A.S. and W.R. performed and contributed to the analysis of tissue pathology. T.J.M. performed surgery, contributed to study conception-design and analysis. K.R. contributed to the conception, design, analyzed/interpreted data and supervised the study. K.R., A.K., J.B. and E.R. wrote the manuscript with input from all authors.

\section{Competing interests}

The authors declare no competing interests.

\section{Additional information}

Supplementary information is available for this paper at https://doi.org/10.1038/s41467 020-15955-w.

Correspondence and requests for materials should be addressed to K.R.

Peer review information Nature Communications thanks Motoyasu Saji, Matthias Trost and the other, anonymous, reviewer for their contribution to the peer review of this work. Peer reviewer reports are available.

Reprints and permission information is available at http://www.nature.com/reprints

Publisher's note Springer Nature remains neutral with regard to jurisdictional claims in published maps and institutional affiliations. 
(c) (i) Open Access This article is licensed under a Creative Commons Attribution 4.0 International License, which permits use, sharing, adaptation, distribution and reproduction in any medium or format, as long as you give appropriate credit to the original author(s) and the source, provide a link to the Creative Commons license, and indicate if changes were made. The images or other third party material in this article are included in the article's Creative Commons license, unless indicated otherwise in a credit line to the material. If material is not included in the article's Creative Commons license and your intended use is not permitted by statutory regulation or exceeds the permitted use, you will need to obtain permission directly from the copyright holder. To view a copy of this license, visit http://creativecommons.org/ licenses/by/4.0/.

(C) The Author(s) 2020 